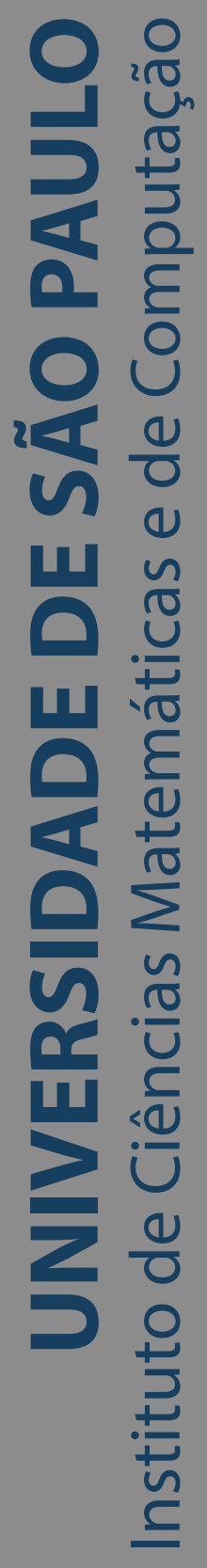

\title{
Topics in Poisson Geometry
}

\section{Murilo do Nascimento Luiz}

Dissertação de Mestrado do Programa de Pós-Graduação em Matemática (PPG-Mat) 

SERVIÇO DE PÓS-GRADUAÇÃO DO ICMC-USP

Data de Depósito:

Assinatura:

\section{Murilo do Nascimento Luiz}

\section{Topics in Poisson Geometry}

Dissertation submitted to the Institute of Mathematics and Computer Sciences - ICMC-USP - in accordance with the requirements of the Mathematics Graduate Program, for the degree of Master in Science. FINAL VERSION

Concentration Area: Mathematics

Advisor: Prof. Dr. Igor Mencattini 
Ficha catalográfica elaborada pela Biblioteca Prof. Achille Bassi e Seção Técnica de Informática, ICMC/USP, com os dados inseridos pelo(a) autor(a)

L953t

Luiz, Murilo do Nascimento

Topics in Poisson Geometry / Murilo do

Nascimento Luiz; orientador Igor Mencattini. -- São Carlos, 2020 .

$78 \mathrm{p}$.

Dissertação (Mestrado - Programa de Pós-Graduação em Matemática) -- Instituto de Ciências Matemáticas e de Computação, Universidade de São Paulo, 2020.

1. Poisson Geometry. 2. Lie Algebroid. 3. Lie Groupoid. 4. Lineatization. I. Mencattini, Igor, orient. II. Título.

Bibliotecários responsáveis pela estrutura de catalogação da publicação de acordo com a AACR2: Gláucia Maria Saia Cristianini - CRB - 8/4938

Juliana de Souza Moraes - CRB - 8/6176 


\section{Murilo do Nascimento Luiz}

\section{Tópicos em Geometria de Poisson}

Dissertação apresentada ao Instituto de Ciências Matemáticas e de Computação - ICMC-USP, como parte dos requisitos para obtenção do título de Mestre em Ciências - Matemática. VERSÃO REVISADA

Área de Concentração: Matemática

Orientadora: Prof. Dr. Igor Mencattini

USP - São Carlos

Fevereiro de 2020 

Este trabalho é dedicado a minha família, que sempre me apoiou e foi um refúgio para os momentos mais difíceis. 

I would like to and I really think that I have to do the acknowledgement in Portuguese, mainly because it is my native language and because my family, who have supported me all those year would not going to understand what they were part.

Primeiramente, eu gostaria de agradecer a minha família, sem a qual nada teria sido possível. Agradeço imensamente a meus pais, João Rosilene. Meus estudos só foram possíveis por causa de todo o suporte que vocês me deram. Agradeço também a Mayara e a Mariana, minhas irmãs queridas que sempre me apoiaram.

Agradeço imensamente também a meu orientador, professor Doutor Igor Mencattini, por todos os encontros e ensinamentos ao longo desses anos. Muito obrigado por toda a paciência e por nunca ter desistido de mim.

Ao Victor, gostaria de dizer muito obrigado por ter sido um amigo leal em todas as horas. Não teria conseguido enfrentar muitas delas sem seu apoio. Obrigado por estar presente nos momentos bons e ruins. Conte comigo para qualquer coisa.

Gostaria de fazer um agradecimento especial para o Ian, por toda a amizade que tivemos durante a graduação e que se estende até os dias de hoje. Também faço um agradecimento para outro amigo que carrego da graduação, Iago. Obrigado por todas as discussões e "rolês". Os momentos que dividi com vocês foram essenciais para a construção de quem eu sou.

Agradeço a Sthéffany e a Érika por todos os bons momentos durante o mestrado, sejam eles tomando um café existencial no meio das tardes, seja perdendo o controle da vida e indo comer fast food de madrugada, ou então cozinhando comidas muito gostosas.

Outra amigo bastante querido e essencial durante todos esses anos tem sido o Uirá. Muito obrigado pelo ótimo amigo que você é, por toda a matemática que me ensinou e que pudemos discutir.

Ana, Breno, Clarissa, Guilherme, Hugo, João, Lemos e Rafael, os amigos cujos laços foram criados e/ou estreitados pela coexistência na pós, compartilhando sala, cafés, discussões e momentos de reflexão, meu mais sincero 'muito obrigado'.

O presente trabalho foi realizado com apoio da Coordenação de Aperfeiçoamento de Pessoal de Nível Superior - Brasil (CAPES) - Código de Financiamento 001 

"Sharing tea with a fascinating stranger is one of life's true delights." (Iroh, Episode 2.08 "The Chase")

"There are good reasons why the theorems should all be easy and the definitions hard." (Michael Spivak, "Calculus on Manifolds") 



\section{RESUMO}

LUIZ, M. N. Tópicos em Geometria de Poisson. 2020. 78 p. Dissertação (Mestrado em Ciências - Matemática) - Instituto de Ciências Matemáticas e de Computação, Universidade de São Paulo, São Carlos - SP, 2020.

Este trabalho é uma revisão bibliografica nas areas de geometria de Poisson e teoria de grupoides e algebroides de Lie. Como resultado final desta interação apresentamos a abordagem geometrica para o teorema de linearização de Conn para estruturas de Poisson apresentada em [Crainic and Fernandes 2011].

Palavras-chave: Geometria de Poisson. Grupoides de Lie. Algebroides de Lie. Linearização. 



\section{ABSTRACT}

LUIZ, M. N. Topics in Poisson Geometry. 2020. 78 p. Dissertação (Mestrado em Ciências Matemática) - Instituto de Ciências Matemáticas e de Computação, Universidade de São Paulo, São Carlos - SP, 2020.

This monograph is a review of Poisson geometry, Lie groupoids and Lie algebroids theory and they relations. We present a summary of the main known results in this areas and as a return of the striking connection of this topics, we present the geometric approach to Conn's linearization theorem present in [Crainic and Fernandes 2011].

Keywords: Poisson Geometry. Lie Groupoids. Lie Algebroids. Linearization. 

INTRODUCTION ..................... 17

POISSON GEOMETRY . . . . . . . . . . . . . . . . . . 19

$2.1 \quad$ Poisson manifold . . . . . . . . . . . . . . . . . . . 19

2.2 The Schouten-Nijenhuis bracket and Poisson cohomology . . . . . 25

2.2.1 Lower degree computations . . . . . . . . . . . . . . . 28

$2.3 \quad$ Examples . . . . . . . . . . . . . . . . . . 29

2.3.1 Symplectic manifolds . . . . . . . . . . . . . . . . . . . . 29

2.3.2 The Lie-Poisson structure on $\mathfrak{g}^{*} \ldots \ldots \ldots$. . . . . . . . . . . . 32

2.3.3 The tangent lifts of a Poisson structure . . . . . . . . . . . . 33

$2.4 \quad$ The Poisson category . . . . . . . . . . . . . . . . 34

$2.5 \quad$ The splitting theorem for Poisson manifolds . . . . . . . . . . 36

$2.6 \quad$ Symplectic foliation . . . . . . . . . . . . . . . 38

2.6.1 Singular foliations and singular distributions . . . . . . . . . . . 39

2.6.2 The symplectic foliation of a Poisson manifold . . . . . . . . . . . . 41

2.6.3 The symplectic foliation of a linear Poisson structure . . . . . . . . . 43

2.7 Isotropy Lie algebra . . . . . . . . . . . . . . . . . . 44

$2.8 \quad$ Complete Poisson manifold . . . . . . . . . . . . . . 45

$2.9 \quad$ Symplectic realization . . . . . . . . . . . . . 46

3 LIE ALGEBROID AND LIE GROUPOID . . . . . . . . . . . . . 49

$3.1 \quad$ Lie algebroid . . . . . . . . . . . . . . 49

3.2 Relation between Lie algebroid theory and Poisson geometry . . . . 52

3.2.1 Isotropy algebras and characteristic foliation of a Lie algebroid . . . 55

$3.2 .2 \quad$ Splitting theorem . . . . . . . . . . . . . . . . 56

3.2.3 Lie algebroid cohomology . . . . . . . . . . . . . 58

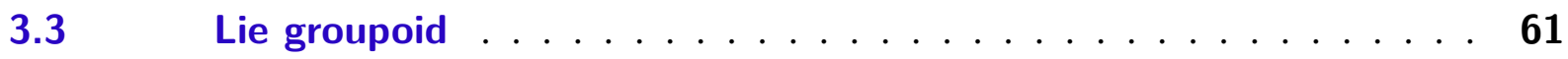

3.3.1 The Lie algebroid of a Lie groupoid . . . . . . . . . . . . . . . . . . 64

$3.4 \quad$ Integrability problem .................. 65

$3.5 \quad$ Lie groupoid cohomology . . . . . . . . . . . . . . 67

4 CONN'S LINEARIZATION THEOREM . . . . . . . . . 71

4.1 Compact Lie Algebras . . . . . . . . . . . . . . 72

$4.2 \quad$ Reduction to a cohomological condition . . . . . . . . . . 73 


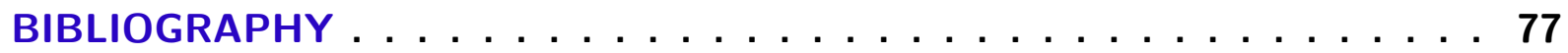


CHAPTER

1

INTRODUCTION

The evolution of a Hamiltonian mechanical system takes place in the so called phasespace which is modeled by a symplectic manifold $(M, \omega)$ whose dimension is equal to number of degrees of freedom of the sytem. The equations describing the time evolution are the so called Hamilton's equations which, in the Darboux's coordinates $\left(q^{1}, \ldots, q^{m}, p_{1}, \ldots, p_{m}\right)$, assume the following form

$$
\begin{aligned}
\dot{q}^{i} & =\frac{\partial h}{\partial p_{i}} \\
\dot{p}_{i} & =-\frac{\partial h}{\partial q^{i}}
\end{aligned}
$$

for all $i \in 1, \ldots, m$. The function $h \in C^{\infty}(M)$ is called the Hamiltonian of the mechanical system and it plays the role of the total energy when the system is not dissipative. Defining

$$
\{f, g\}:=\sum_{i=1}^{n} \frac{\partial f}{\partial p_{i}} \frac{\partial g}{\partial q^{i}}-\frac{\partial f}{\partial q^{i}} \frac{\partial g}{\partial p_{i}},
$$

for all $f, g \in C^{\infty}(M)$, and given $h \in C^{\infty}(M)$ the Hamilton's equations can be written as

$$
\begin{aligned}
\dot{q}_{i} & =\left\{h, q_{i}\right\} \\
\dot{p}_{i} & =\left\{h, p_{i}\right\}
\end{aligned}
$$

for all $i=1, \ldots, m$.

This bracket satisfies the following proprieties

1. Skew-symmetry: $\{f, g\}=-\{g, f\}$,

2. Bilinearity: $\{a f+b g, h\}=a\{f, h\}+b\{g, h\}$,

3. Jacobi identity: $\{\{f, g\}, h\}+\{\{g, h\}, f\}+\{\{h, f\}, g\}=0$,

4. Leibniz rule: $\{f, g h\}=g\{f, h\}+\{f, g\} h$, 
for all $f, g, h \in C^{\infty}(M)$ Abstracting these proprieties one can introduce the concept Poisson manifold, e.g. a pair $(M,\{\cdot, \cdot\})$ where $M$ is a smooth manifold and $\{\cdot, \cdot\}$ is a bracket on $C^{\infty}(M)$ satisfies the previous proprieties. Poisson structures appear in many different areas of mathematics, for example dynamical systems, differential geometry, abstract algebra and representation theory. In all of these areas the possibility to approximate, in a suitable sense, a Poisson structure with a linear one is of the greatest importance and for this reason the results describing the possible normal forms theorems for these geometric structures, play a fundamental role in Poisson geometry.

The main goal of this dissertation is to present a summary of the main known results in this area, emphasizing the geometrical perspective.

This monograph is organized as follows. In Chapter 2 we introduce the basics of the theory of Poisson manifolds like the Weinstein splitting theorem, describing the local structure of Poisson manifolds and implying the existence of the symplectic foliation. In Chapter 3 we present a summary of the theory of Lie groupoids and Lie algebroids and we introduce the key tools to develop the theory presented in the following chapter. Following the Spivak's philosophy, saying that in a theory we must make the hard work on the definitions and win suitable proves, in Chapter 4 we present a geometric approach to Conn's linearization theorem as explained in [Crainic and Fernandes 2011]. Throughout this work, unless stated otherwise, we assume that all the manifolds are smooth. 
CHAPTER

2

POISSON GEOMETRY

Proposed by Poisson as a tool for Lagrangian mechanics, and studied by Jacobi and Lie, the Poisson bracket has connections with different areas such as Lie groups and Lie algebras, classical mechanics, singularity theory, quantum mechanics and others [Weinstein 1998].

The goal of this chapter is to introduce the Poison Geometry. First, we present the basics facts and definitions. In the Section 2.2 we introduce the Schouten bracket, use it to define the cohomology of Poisson manifolds and compute the lower degree groups. Next, we present some important examples like symplectic manifold and the linear Poisson bracket. In the Section 2.4 we define the notion of Poisson morphism and define the Poisson category, showing that the Hamiltonian vector field, defined from a $f \in C^{\infty} M$, is invariant under Poisson morphism. In Section 2.5 we present the main result in local theory of Poisson manifolds, the Weinstein's splitting theorem. On the next section, we present the main result about the global structures of a Poisosn manifold, the symplectic foliantion. At last, in the final three sections we define the isotropy algebra, the concept of complet Poisosn map and symplectic realization.

\subsection{Poisson manifold}

Definition 1. . A smooth Poisson structure on a finite dimensional manifold $M$ is an $\mathbb{R}$ bilinear skew-symmetric operation $C^{\infty}(M) \times C^{\infty}(M) \rightarrow C^{\infty}(M),(f, g) \mapsto\{f, g\}$ defined on the algebra of the real-valued smooth functions on M satisfying, the:

1. Jacobi identity

$$
\{\{f, g\}, h\}+\{\{g, h\}, f\}+\{\{h, f\}, g\}=0,
$$

2. Leibniz identity

$$
\{f, g h\}=g\{f, h\}+\{f, g\} h,
$$

for all $f, g, h \in C^{\infty}(M)$. 
We call the pair $(M,\{\cdot, \cdot\})$ a Poisson Manifold. One can check that any manifold with the bracket $\{f, g\}=0$ for all $f, g \in C^{\infty}(M)$ is a Poisson manifold.

An important consequence of the $\mathbb{R}$-bilinearity and the Leibniz identity is that the Poisson bracket is a local operation, i.e., $\{f, g\}(x)$ depends on the values of the functions $f, g$ in a neighborhood of $x$. Formally speaking, we have:

Proposition 2. Let $f, f^{\prime} \in C^{\infty}(M)$ and $x \in M$, and suppose that there is $U \subset M$, an open neighborhood of $x$, such that $\left.f\right|_{U}=\left.f^{\prime}\right|_{U}$. Then

$$
\{f, g\}(x)=\left\{f^{\prime}, g\right\}(x), \forall g \in C^{\infty}(M) .
$$

Proof. Let $\alpha \in C^{\infty}(M)$ be a function such that $\alpha(x)=1$ and $\operatorname{supp}(\alpha)=\{x \in M \mid \alpha(x) \neq 0\} \subset U$, then, $\alpha\left(f-f^{\prime}\right)=0$. Let $g \in C^{\infty}(M)$, thus,

$$
\begin{aligned}
0 & =\left\{g, \alpha\left(f-f^{\prime}\right)\right\}(x)=\alpha(x)\left\{g,\left(f-f^{\prime}\right)\right\}(x)+\{g, \alpha\}(x)\left(f-f^{\prime}\right)(x) \\
& =\left\{g,\left(f-f^{\prime}\right)\right\}=\{g, f\}(x)-\left\{g, f^{\prime}\right\}(x) .
\end{aligned}
$$

Thanks to proposition (2), we can restrict the Poisson bracket to an open set $U \subset M$. For $f, g \in C^{\infty}(U)$, take a compact subset $K$ of $U$ and $\alpha \in C^{\infty}(M)$ such that $\operatorname{supp}(\alpha) \subset K$. Hence, we have two global functions $\alpha f, \alpha g \in C^{\infty}(M)$, satisfying $\left.\alpha f\right|_{K}=\left.f\right|_{K}$ and $\left.\alpha g\right|_{K}=\left.g\right|_{K}$. So, we define

$$
\left.\{f, g\}\right|_{U}:=\{\alpha f, \alpha g\}
$$

By proposition (2) the definition of $\left.\{f, g\}\right|_{U}$ does not depend on the function $\alpha$.

There is an important subalgebra of the Lie algebra of smooth vector fields: the Hamiltonian vector fields.

Definition 3. Given $f$ a smooth function, we define the Hamiltonian vector field of $f$, denoted by $X_{f}$, as the unique vector field that satisfies

$$
X_{f}(g):=\{f, g\}, \forall g \in C^{\infty}(M) .
$$

It is clear that, by skew-symmetry, we have

$$
X_{f}(g)=\{f, g\}=-X_{g}(f) \text {. }
$$

The following lemma ensures that the subset of a Hamiltonian vector field is truly a Lie subalgebra. 
Proposition 4. The map $f \rightarrow X_{f}$ is a homomorphism from the Lie algebra $C^{\infty}(M)$ of smooth functions under the Poisson bracket to the Lie algebra of smooth vector fields under the usual Lie bracket. In other words,

$$
\left[X_{f}, X_{g}\right]=X_{\{f, g\}}
$$

Proof. For any $f, g, h \in C^{\infty}(M)$ we have

$$
\begin{aligned}
{\left[X_{f}, X_{g}\right] h } & =X_{f}\left(X_{g}(h)\right)-X_{g}\left(X_{f}(h)\right) \\
& =\{f,\{g, h\}\}-\{g,\{f, h\}\} \\
& =\{f,\{g, h\}\}+\{g,\{h, f\}\} .
\end{aligned}
$$

By the Jacobi's identity, we have

$$
\begin{aligned}
{\left[X_{f}, X_{g}\right] h } & =\{\{f, g\}, h\} \\
& =X_{\{f, g\}}(h) .
\end{aligned}
$$

Since $h$ is arbitrary, it means that $\left[X_{f}, X_{g}\right]=X_{\{f, g\}}$.

Proposition 5. Let $\varphi_{t}$ be the flow of a vector field $X \in \mathfrak{X}(M)$ and let $h \in C^{\infty}(M)$. Then

1. Let $U \subset M$ be an open subset. For all $f \in C^{\infty}(U)$,

$$
\frac{d}{d t}\left(f \circ \varphi_{t}\right)=\{h, f\} \circ \varphi_{t}=\left\{h, f \circ \varphi_{t}\right\}
$$

if, and only if, $\varphi_{t}$ is the flow of $X_{h}$.

2. If $\varphi_{t}$ is the flow of $X_{h}$, then $h \circ \varphi_{t}=h$.

Proof. Let $x \in M$, then

$$
\begin{aligned}
& \frac{d}{d t}\left(f \circ \varphi_{t}(x)\right)=d f_{\left(\varphi_{t}(x)\right)}\left(\frac{d}{d t}\left(\varphi_{t}(x)\right)\right) \\
& \{h, f\}\left(\varphi_{t}(x)\right)=d f_{\left(\varphi_{t}(x)\right)}\left(X_{h}\left(\varphi_{t}(x)\right)\right)
\end{aligned}
$$

the left-hand side of two equations are equal for any $f \in C^{\infty}(U)$, if, and only if,

$$
\frac{d}{d t}\left(\varphi_{t}(x)\right)=X_{h}\left(\varphi_{t}(x)\right)
$$

On the other hand, if $\varphi_{t}$ is the flow of $X_{h}$, then

$$
X_{h}\left(\varphi_{t}(x)\right)=d_{x} \varphi_{t}\left(X_{h}(x)\right)
$$

the chain rule implies that

$$
\begin{aligned}
\frac{d}{d t}\left(f\left(\varphi_{t}(x)\right)\right) & =d f_{\left(\varphi_{t}(x)\right)}\left(X_{h}\left(\varphi_{t}(x)\right)\right) \\
& =d f_{\left(\varphi_{t}(x)\right)}\left(T_{x} \varphi_{t}\left(X_{h}(x)\right)\right) \\
& =d\left(f \circ \varphi_{t}\right)_{x}\left(X_{h}(x)\right) \\
& =\left\{f \circ \varphi_{t}, h\right\}(x) .
\end{aligned}
$$

The second part is the first one when $h=f$. 
And we have the following corollary.

Corollary 6. Let $g, h \in C^{\infty}(M)$ such that $\{g, h\}=0$, then $g$ is constant along the integral curves of $X_{h}$.

Is it possible to understand the Poisson bracket, $\{\cdot, \cdot\}$, as a $C^{\infty}$ tensor field $\Pi \in \mathfrak{X}^{2}(M)$ $\Pi: T^{*} M \times T^{*} M \rightarrow \mathbb{R}$ such that

$$
\Pi_{x}\left(d f_{x}, d g_{x}\right):=\{f, g\}(x)
$$

In a local coordinate $\left(x^{i}\right), \Pi$ has the following representation

$$
\{f, g\}=\Pi(d f, d g)=\sum_{i, j} \Pi^{i j} \frac{\partial f}{\partial x^{i}} \frac{\partial g}{\partial x^{j}} \forall f, g \in C^{\infty}(M),
$$

where $\Pi^{i j}=\left\{x^{i}, x^{j}\right\}$. We call $\Pi$ the Poisson bivector of $(M,\{\cdot, \cdot\})$.

Conversely, given a bivector field $\Pi$, we obtain a skew-symmetric bracket on $C^{\infty}(M)$ by

$$
\{f, g\}:=\Pi(d f, d g)
$$

Since the above bracket is a bi-derivation, to ensure that it is Poisson, it is just necessary to find the conditions which ensure that $\{\cdot, \cdot\}$ satisfies the Jacobi identity.

For example, in terms of the Lie derivative of $\Pi$, we can write:

$$
\begin{aligned}
\mathscr{L}_{X_{h}} \Pi(d f, d g) & =X_{h}(\{f, g\})-\left\{X_{h}(f), g\right\}-\left\{f, X_{h}(g)\right\} \\
& =\{\{f, g\}, h\}-\{\{f, h\}, g\}-\{f,\{g, h\}\} \\
& =\{\{f, g\}, h\}+\{\{g, h\}, f\}+\{\{h, f\}, g\} .
\end{aligned}
$$

Thus, the Jacobi identity for $\{\cdot, \cdot\}$ can be replaced by $\mathscr{L}_{X_{f}} \Pi=0$, for all $f$.

Another way is in term of a 3-vector field, called Jac.

Proposition 7. For any smooth 2-vector field $\Pi$, we can associate to it a 3 -vector field defined by

$$
\operatorname{Jac}_{\Pi}(f, g, h)=\{\{f, g\}, h\}+\{\{g, h\}, f\}+\{\{h, f\}, g\} .
$$

Proof. Since $\{\cdot, \cdot\}$ is $\mathbb{R}$-bilinear, so is Jac $\mathbb{R}$-trilinear. The cyclic sum and skew-symmetry of the bracket gives the skew-symmetry of Jac. We just have to check if it satisfies the Leibniz identity. Indeed,

$$
\begin{aligned}
& \left\{\left\{f_{1} f_{2}, g\right\}, h\right\}=f_{1}\left\{\left\{f_{2}, g\right\}, h\right\}+\left\{f_{2}, g\right\}\left\{f_{1}, h\right\}+f_{2}\left\{\left\{f_{1}, g\right\}, h\right\}+\left\{f_{1}, g\right\}\left\{f_{2}, h\right\} ; \\
& \left\{\{g, h\}, f_{1} f_{2}\right\}=f_{1}\left\{\{g, h\} f_{2}\right\}+f_{2}\left\{\{g, h\} f_{1}\right\} ; \\
& \left\{\left\{h, f_{1} f_{2}\right\}, g\right\}=f_{1}\left\{\left\{h, f_{2}\right\}, g\right\}+\left\{f_{1}, g\right\}\left\{h, f_{2}\right\}+f_{2}\left\{\left\{h, f_{1}\right\}, g\right\}+\left\{f_{2}, g\right\}\left\{h, f_{1}\right\} .
\end{aligned}
$$


and we have that,

$$
\begin{aligned}
\operatorname{Jac}\left(f_{1} f_{2}, g, h\right)= & \left.f_{1}\left(\left\{\left\{f_{2}, g\right\}, h\right\}+\left\{\{g, h\} f_{2}\right\}+\left\{h, f_{2}\right\}, g\right\}\right) \\
& +f_{2}\left(\left\{\left\{f_{1}, g\right\}, h\right\}+\left\{\{g, h\} f_{1}\right\}+\left\{\left\{h, f_{1}\right\}, g\right\}\right) \\
= & f_{1} \operatorname{Jac}\left(f_{2}, g, h\right)+\operatorname{Jac}\left(f_{1}, g, h\right) f_{2}
\end{aligned}
$$

In local coordinates $\left(x^{1}, \ldots, x^{n}\right) \mathrm{Jac}_{\Pi}$ can be write as

$$
\operatorname{Jac}_{\Pi}=\sum_{i, j, k}\left(\oint_{i j k} \sum_{s} \frac{\partial \Pi^{i, j}}{\partial x^{s}} \Pi^{s, k}\right) \frac{\partial}{\partial x^{i}} \wedge \frac{\partial}{\partial x^{j}} \wedge \frac{\partial}{\partial x^{k}},
$$

where $\oint_{i j k} a_{i j k}$ means the cyclic sum $a_{i j k}+a_{j k i}+a_{k i j}$.

Thus, the following statements are equivalents

- $\{\cdot, \cdot\}$ is a Poisson bracket;

- $\left[X_{f}, X_{g}\right]=X_{\{f, g\}}$ for all $f, g$;

- $\mathscr{L}_{X_{f}} \Pi=0$ for all $f$;

- $\oint_{i j k} \sum_{s} \frac{\partial \Pi_{i, j}}{\partial x_{s}} \Pi_{s, k}=0$ for all $i, j, k$.

A Poisson structure $\Pi$ on $M$, defines a natural vector bundle morphism,

$$
\#: T^{*} M \rightarrow T M,
$$

which maps each covector $\alpha \in T_{x}^{*} M$ to the vector $\#(\alpha) \in T_{x} M$ such that

$$
\langle\alpha \wedge \beta, \Pi\rangle_{x}=\left\langle\beta, \#_{x}(\alpha)\right\rangle_{x}, \beta \in T_{x}^{*} M
$$

where the $\langle\cdot, \cdot\rangle_{x}$ is the pering between $T_{x}^{*} M$ and $T_{x} M$.

Definition 8. Let $(M, \Pi)$ be a Poisson manifold and $x \in M$. The characteristic space at $x$ is $C_{x}:=\operatorname{Im} \#_{x}$. The dimension of $C_{x}$ is called the rank of $\Pi$ at $x$. We call $x$ a regular point of $(M, \Pi)$ if there is a neighborhood $U$ of $x$ such that $\operatorname{Rank}\left(\Pi_{x}\right)=\operatorname{Rank}\left(\Pi_{y}\right)$ for every $y \in U$. Otherwise, we call $x$ a singular point of $(M, \Pi)$. If $\operatorname{Rank}\left(\Pi_{x}\right)$ does not depend on $\mathrm{x}$, then $\Pi$ is called a regular Poisson structure. We call $M_{\text {reg }}=\{x \in M \mid x$ is a regular point $\}$ the set of regular points and we call $M_{\text {sig }}=\{x \in M \mid x$ is a singular point $\}$ the set of singular points.

Proposition 9. Let $(M, \Pi)$ be a Poisson manifold. For every $x \in M$ the rank of $\Pi$ at $x$ is an even number. 
Proof. Suppose $\operatorname{dim} M=d$ and let $(U, \varphi)$ be a coordinate neighborhood of $x \in M$. On $U$, the bivector field can be written as

$$
\Pi=\sum_{1 \leqslant i<j \leqslant d}\left\{y^{i}, y^{j}\right\} \frac{\partial}{\partial y^{i}} \wedge \frac{\partial}{\partial y^{j}} .
$$

Evaluating the Poisson structure on $x \in U$, we get a skew-symmetric bilinear map $\Pi_{x}: T_{x}^{*} M \times$ $T_{x}^{*} M \rightarrow \mathbb{R}$.

Let $U:=\left\{\mu \in T_{x}^{*} M \mid \Pi_{x}(\mu, v)=0 \forall v \in T_{x}^{*} M\right\}$. Choose a complementary space $W$ in $T_{x}^{*} M$

$$
T_{x}^{*} M=U \oplus W
$$

Take any nonzero $q_{1} \in W$. So, there is $p_{1}$ such that $\Pi_{x}\left(q_{1}, p_{1}\right) \neq 0$. Let

$$
\begin{gathered}
W_{1}=\operatorname{span}\left\{p_{1}, q_{1}\right\} \\
W_{1}^{\prime}=\left\{w \in W \mid \pi_{x}(w, v)=0 \quad \forall v \in W_{1}\right\} .
\end{gathered}
$$

So, we have that $W_{1} \cap W_{1}^{\prime}=\{0\}$ and $W=W_{1} \oplus W_{1}^{\prime}$. Repeating this process we get

$$
V=U \oplus W_{1} \oplus W_{2} \oplus \ldots \oplus W_{n}
$$

Thus the rank of $\Pi_{x}$ is $2 n$.

The rank of a Poisson manifold is not necessarily continuous with respect to $x \in M$, however, it varies properly with $x$. Here, the meaning of "properly" follows the next definition

Definition 10. Let $X$ be a topological space and $f: X \rightarrow \mathbb{N}$. We say that $f$ is lower semicontinuous at $x \in X$ if there is a neighborhood $V$ of $x$ such that $f(y) \geq f(x)$ for all $y \in V$.

This definition is not the usual general definition of lower semi-continuity, but it is an adaptation to our context. Equipped with the above definition, we can prove the following proposition.

Proposition 11. The function $\operatorname{Rank}_{\Pi}: M \rightarrow \mathbb{N}$ defined by $x \mapsto \operatorname{Rank}\left(\Pi_{x}\right)$ is lower semi-continuous.

Proof. Take some local coordinates $\left(x^{1}, \ldots, x^{n}\right)$ around $x \in M$. If the $\operatorname{Rank}(x)=2 n$ the matrix $\Pi^{i j}(x)$ had Rank $2 n$, there wold be a submatrix, $A$, of $\# \Pi^{i, j}$ of size $2 n \times 2 n$ with non-zero determinant. Since the determinant is a continuous function, there is a neighborhood $U$ of $x$ such that the determinant of $A(y)$ must not vanish for all $y \in U$, and the $\operatorname{Rank}_{\Pi}$ is at least $2 n$ on this neighborhood.

Using the above proposition, we can prove the following one

Proposition 12. the set $M_{\text {reg }}$, of regular points of a Poisson Manifold, is a dense subset of $M$ and the set $M_{\text {sing }}$, of singular points, is a closed nowhere dense subset of $M$. 
Proof. From the definition, $M_{\text {reg }}$ is a union of open sets, therefore open. Since $M_{\text {sing }}$ is the complement of $M_{\text {reg }}$, it is closed.

To prove that $M_{\text {reg }}$ is a dense subset, suppose that $x_{0} \in M$ is a singular point and let $V$ be a neighborhood of $x_{0}$. Since $\operatorname{Rank}(y) \in\{0,1, \cdots, n\}$ there is some $y_{0} \in V$ such that $\operatorname{Rank}(y)=n$ and then, by the lower semi-continuity, $y_{0}$ is a regular point. Since $M_{r e g}$ is a open dense subset, its complement, $M_{\text {sing }}$, is a closed nowhere dense.

\subsection{The Schouten-Nijenhuis bracket and Poisson coho- mology}

This section follows closely the formulation presented in [Vaisman 2012] and [Dufour and Zung 2006]. Aiming to define the cohomology of Poisson manifold, we present the SchoutenNijenhuis bracket. This operation is an extension of the Lie bracket of vector fields to the multivector fields on a smooth manifold.

The following theorem is the key to define the Schouten-Nijenhuis bracket.

Theorem 13. There is a unique bilinear operation $[\cdot, \cdot]: \mathfrak{X}^{\bullet}(M) \times \mathfrak{X}^{\bullet}(M) \rightarrow \mathfrak{X}^{\bullet}(M)$, called the Schouten bracket, that satisfies the following properties:

I. For all $f, g \in C^{\infty}(M),[f, g]=0$;

II. For all $A, B, C \in \mathfrak{X}^{\bullet}(M)$ then

$$
[A, B \wedge C]=[A, B] \wedge C+(-1)^{(\operatorname{deg}(A)-1) \operatorname{deg}(B)} B \wedge[A, C]
$$

and $\operatorname{deg}([A, B])=\operatorname{deg}(A)+\operatorname{deg}(B)-1$;

III. For functions $f, g \in C^{\infty}(M)$ and vector fields $X, Y \in \mathfrak{X}(M)$, the Schouten bracket coincides with the Lie derivative, i.e.,

a) $[X, f]=X(f)$;

b) $[X, Y]$ is the usual Lie bracket for vector fields.

IV. $[A, B]=-(-1)^{(\operatorname{deg}(A)-1)(\operatorname{deg}(B)-1)}[B, A]$;

V. $(-1)^{\operatorname{deg}(A) \operatorname{deg}(C)}[[A, B], C]+(-1)^{\operatorname{deg}(B) \operatorname{deg}(A)}[[B, C], A]+(-1)^{\operatorname{deg}(C) \operatorname{deg}(B)}[[C, A], B]=0$.

where $\operatorname{deg}(A)$ is the degree of $A$.

This operation is defined by

$$
\left[X_{1} \wedge \ldots \wedge X_{p}, B\right]=\sum_{i=1}^{p}(-1)^{i+1} X_{1} \wedge \ldots \wedge \hat{X}_{i} \wedge X_{p} \wedge\left[X_{i}, B\right]
$$


where $X_{1}, \ldots, X_{p} \in \mathfrak{X}(M),\left[X_{i}, A\right]:=\mathscr{L}_{X_{i}}(A)$, and the hat, as usual, denotes that the $X_{i}$ is not present in the wedge product.

Using the above definition and the fact that the Schouten bracket must be a local operator, Vaisman proved the theorem (13) in [Vaisman 2012].

One can get another characterization to the Schouten bracket using the following lemma

Lemma 14. For any $A \in \mathfrak{X}^{a}(M), B \in \mathfrak{X}^{b}(M), \omega \in \Omega^{a+b-1}(M)$

$$
\begin{aligned}
i_{[A, B]}(\omega)= & (-1)^{(a-1)(b-1)} i_{A}\left(d\left(i_{B}(\omega)\right)\right) \\
& +(-1)^{a} i_{A \wedge B}(d(\omega))+(-1)^{b} d\left(i_{A \wedge B}(\omega)\right) .
\end{aligned}
$$

In local coordinates $\left(y^{1}, \ldots, y^{n}\right)$, any $A \in \wedge^{a} T M$, with $a \geq 1$, is given by

$$
A=\sum_{i_{1}<\ldots<i_{a}} A^{i_{1}, \ldots, i_{a}} \frac{\partial}{\partial y^{i_{1}}} \wedge \ldots \wedge \frac{\partial}{\partial y^{i_{a}}} .
$$

When $B=f \in C^{\infty}(M)$ is a smooth function, the equation (2.2.2) is given in coordinates by

$$
[A, f]=\sum_{s=1}^{a}(-1)^{s+1} \sum_{i_{1}<\ldots<s<\ldots<i_{a}} A^{i_{1}, \ldots, i_{a}} \frac{\partial f}{\partial y^{s}} \frac{\partial}{\partial y^{i_{1}}} \wedge \ldots \wedge \frac{\hat{\partial}}{\partial y^{s}} \wedge \frac{\partial}{\partial y^{i_{a}}} .
$$

Aiming to express the Schouten bracket in that local coordinate for two arbitrary multivector fields, we should put the expansion (2.2.3) in the equation (2.2.2).

$$
\begin{aligned}
{[A, B]=} & \sum_{s=1}^{a} \sum_{\left(i_{1}<\ldots<s<\ldots<i_{a}\right)} \sum_{\left(j_{1}<\ldots<i_{b}\right)} A^{\left(i_{1}, \ldots, i_{a}\right)} \frac{\partial B^{\left(j_{1}, \ldots, j_{b}\right)}}{\partial y^{s}} \frac{\partial}{\partial y^{i_{1}}} \wedge \ldots \wedge \frac{\hat{\partial}}{\partial y^{s}} \wedge \frac{\partial}{\partial y^{i_{a}}} \\
& \wedge \frac{\partial}{\partial y^{j_{1}}} \wedge \ldots \wedge \frac{\partial}{\partial y^{j_{b}}} \\
& -(-1)^{(a-1)(b-1)} \sum_{s=1}^{a} \sum_{\left(j_{1}<\ldots<s<\ldots<j_{b}\right)} \sum_{\left(i_{1}<\ldots<i_{a}\right)} B^{\left(j_{1}, \ldots, j_{a}\right)} \frac{\partial A^{\left(i_{1}, \ldots, i_{a}\right)}}{\partial y^{s}} \frac{\partial}{\partial y^{j_{1}}} \wedge \ldots \wedge \frac{\hat{\partial}}{\partial y^{s}} \wedge \frac{\partial}{\partial y^{j_{b}}} \\
& \wedge \frac{\partial}{\partial y^{i_{1}}} \wedge \ldots \wedge \frac{\partial}{\partial y^{i_{a}}}
\end{aligned}
$$

Using the expression in coordinates for an arbitrary bivector $\Pi=\sum_{i<j} \pi^{i, j} \frac{\partial}{\partial y^{i}} \wedge \frac{\partial}{\partial y^{j}}$, along with the previous formula, one can show that

$$
[\Pi, \Pi]=\mathrm{Jac}=\sum_{i, j, k}\left(\oint_{i j k} \sum_{s} \frac{\partial \Pi^{i, j}}{\partial y^{s}} \Pi^{s, k}\right) \frac{\partial}{\partial y^{i}} \wedge \frac{\partial}{\partial y^{j}} \wedge \frac{\partial}{\partial y^{k}} .
$$

In other words:

Proposition 15. A bivector field $\Pi$ is a Poisson bivetor if and only if,

$$
[\Pi, \Pi]=0 .
$$


Since $[\Pi, \Pi]$ is a trivector field, the previous proposition implies that, if $M$ is a two dimensional manifold, then every bivector field $\Pi$ on $M$ is Poisson.

Definition 16. We define the Poisson cohomology as the cohomology of the following complex

$$
\ldots \stackrel{d_{\Pi}}{\longrightarrow} \mathfrak{X}^{p-1}(M) \stackrel{d_{\Pi}}{\longrightarrow} \mathfrak{X}^{p}(M) \stackrel{d_{\Pi}}{\longrightarrow} \mathfrak{X}^{p+1}(M) \stackrel{d_{\Pi}}{\longrightarrow} \ldots
$$

Where $d_{\Pi}(A)=[\Pi, A]$.

It is necessary to show that $d_{\Pi} \circ d_{\Pi}=0$.

Proposition 17. If $\Pi$ is a Poisson tensor, then for every multivector field $A$, we have

$$
[\Pi,[\Pi, A]]=0 .
$$

Proof. Let A be a multivector field of Rank $a$. By the graded Jacobi identity

$$
(-1)^{(a-1)(2-1)}[\Pi,[\Pi, A]]+(-1)^{(2-1)(2-1)}[\Pi,[A, \Pi]]+(-1)^{(a-1)(2-1)}[A,[\Pi, \Pi]]=0 .
$$

But $[A, \Pi]=-(-1)^{(a-1)(2-1)}[\Pi, A]$ and, since $\Pi$ is a Poisson tensor, $[\Pi, \Pi]=0$, thus

$$
(-1)^{(a-1)}[\Pi,[\Pi, A]]+(-1)^{(a+1)}=2(-1)^{(a-1)}[\Pi,[\Pi, A]]=0 .
$$

So, $[\Pi,[\Pi, A]]=0$, as we wished.

The Poisson cohomology groups are given by the quotient

$$
H_{\Pi}^{p}(M)=\frac{\operatorname{Ker}\left(d_{\Pi}: \mathfrak{X}^{p}(M) \rightarrow \mathfrak{X}^{p+1}(M)\right)}{\operatorname{Im}\left(d_{\Pi}: \mathfrak{X}^{p-1}(M) \rightarrow \mathfrak{X}^{p}(M)\right)} .
$$

Furthermore, the Schouten bracket can be used to describe the Hamiltonian vector fields.

Proposition 18. If $(M, \Pi)$ is a Poisson manifold and $f \in C^{\infty}(M)$, then the corresponding Hamiltonian vector field $X_{f}$ satisfies the equation

$$
X_{f}=-[\Pi, f]
$$

Proof. Setting $A$ as a bivector field on the equation (2.2.4), we have that

$$
[\Pi, f]=\sum_{i<j} \pi^{i j} \frac{\partial}{\partial x^{i}} \frac{\partial f}{\partial x^{j}}=-\sum_{i<j} \pi^{i j} \frac{\partial f}{\partial x^{i}} \frac{\partial}{\partial x^{j}}=-X_{f} .
$$




\subsubsection{Lower degree computations}

In general, it is very difficult to compute the $H_{\Pi}$, but one can give some interpretation to the firsts Poisson cohomology groups.

Definition 19. Let $f \in C^{\infty}(M)$ be a smooth function on a Poisson manifold. We say that $f$ is a Casimir function if $X_{f}=0$.

The Proposition (18) shows that the $\operatorname{Ker}\left(d_{\Pi}: C^{\infty}(M) \rightarrow \mathfrak{X}(M)\right)$ is the vector space of Casimir functions and thus $\operatorname{Im}\left(d_{\Pi}: C^{\infty}(M) \rightarrow \mathfrak{X}(M)\right)$ is the set of Hamiltonian vector fields. So the zero Poisson cohomology group $H_{\Pi}^{0}(M)$ is the set of Casimir functions.

Definition 20. Let $X \in \mathfrak{X}(M)$. We call $X$ a Poisson vector field if

$$
\mathscr{L}_{X}(\Pi)=0
$$

For the first Poisson group, we have that the $\operatorname{Ker}\left(d_{\Pi}: \mathfrak{X}(M) \rightarrow \mathfrak{X}^{2}(M)\right)$ is the set of vector field such that $\mathscr{L}_{X}(\Pi)=-[\Pi, X]=0$. So $H_{\Pi}^{1}$ is the quotients of the Poisson vector fields by the Hamiltonian vector fields.

Definition 21. Let $\Pi_{1}, \Pi_{2}$ be two Poisson bi-vectors to a smooth manifold $M$. We say that $\Pi_{1}$ and $\Pi_{2}$ are compatible Poisson structures if $\Pi_{1}+\Pi_{2}$ is a Poisson structure to $M$.

One can rewrite this condition in terms of the Schouter bracket using that

$$
\left[\Pi_{1}+\Pi_{2}, \Pi_{1}+\Pi_{2}\right]=\left[\Pi_{1}, \Pi_{1}\right]+\left[\Pi_{2}, \Pi_{2}\right]+2\left[\Pi_{1}, \Pi_{2}\right]=2\left[\Pi_{1}, \Pi_{2}\right]
$$

So, two Poisson structures are compatible if, and only if,

$$
\left[\Pi_{1}, \Pi_{2}\right]=0
$$

If $\Pi_{1}$ and $\Pi_{2}$ are compatible Poisson structures, so will do $\varepsilon_{1} \Pi_{1}$ and $\varepsilon_{2} \Pi_{2}$.

Let $\Pi(\varepsilon)$ be a smooth family of Poisson structures. Write

$$
\Pi(\varepsilon)=\Pi_{0}+\varepsilon \Pi_{1}+\varepsilon^{2} \Pi_{2} \ldots
$$

as a power series expression. To $\Pi(\varepsilon)$ be a Poisson structure we need that

$$
\begin{aligned}
{[\Pi(\varepsilon), \Pi(\varepsilon)] } & =\left[\Pi_{0}, \Pi_{0}\right]+2 \varepsilon\left[\Pi_{0}, \Pi_{1}\right]+\varepsilon^{2}\left(2\left[\Pi_{0}, \Pi_{2}\right]+\left[\Pi_{1}, \Pi_{1}\right]\right)+\ldots \\
& =0
\end{aligned}
$$

The coefficient $\Pi_{1}$ is called an infinitesimal deformation of $\Pi_{0}$ when

$$
d_{\Pi_{0}}\left(\Pi_{1}\right)=\left[\Pi_{0}, \Pi_{1}\right]=0 .
$$


Since $d^{2}=0$, every $\Pi$ defined by

$$
\Pi_{1}=d_{\Pi_{0}}(X)=\left[\Pi_{0}, V\right]=-\mathscr{L}_{X}\left(\Pi_{0}\right)
$$

is an infinitesimal deformation. Let $\varphi_{t}$ be the flow of $-X$. For each $\mathrm{t}$, the pull-back $\varpi_{t}^{*} \Pi_{0}$ is again a Poisson structure. Furthermore,

$$
\left.\frac{d}{d t} \varphi_{t}^{*} \Pi_{0}\right|_{t=0}=\mathscr{L}_{-X}\left(\Pi_{0}\right) \Pi_{1} .
$$

On this case, we call $\Pi_{1}$ a trivial infinitesimal deformation of $\Pi_{0}$. This deformation is trivial in the sense that $\frac{d}{d t} \varphi_{t}^{*} \Pi_{0}$ are the same Poisson structure up to change of coordinate. So we have that the second Poisson cohomology group is given by

$$
H_{\Pi}^{2}=\frac{\text { infinitesimal deformation of } \Pi}{\text { trivial infinitesimal deformation of } \Pi}
$$

It is possible to extend the definition of \# to forms of any degree by

$$
\#(\omega)\left(\alpha_{1}, \ldots, \alpha_{r}\right)=(-1)^{r}\left(\# \alpha_{1}, \ldots, \# \alpha_{r}\right) .
$$

We can associate the Poisson cohomology with de Rham cohomology by the next proposition.

Proposition 22. For every smooth differential form $\eta$ on a Poisson manifold $M$, we have

$$
d_{\pi}(\# \omega)=\#(d \omega),
$$

were $d_{\pi}$ is the differential operation on the Poisson cohomology and $d$ is the differential operation on de Rham cohomology.

The demonstration of the above proposition can be find in [Dufour and Zung 2006].

\subsection{Examples}

\subsubsection{Symplectic manifolds}

Definition 23. Let $M$ be a manifold. A 2-form $\omega$ on $M$ is called symplectic if it is closed and non-degenerate. We call the pair $(M, \omega)$ a symplectic manifold.

The notion of symplectormorphism is defined as:

Definition 24. Let $\left(M_{1}, \omega_{1}\right)$ and $\left(M_{2}, \omega_{2}\right)$ be a symplectic manifold and let $\varphi: M_{1} \rightarrow M_{2}$ be a diffeomorphism. We call $\varphi$ a symplectomorphism if

$$
\omega_{2}\left(\varphi_{*}(X), \varphi_{*}(Y)\right)=\omega_{1}(X, Y)
$$


For every $f$ smooth function on the symplectic manifold $M$, we can associate a Hamiltonian vector field, $X_{f}$, by the only vector field such that, $i_{X_{f}} \omega=d f$, in other words, for every $Y \in \mathfrak{X}(M)$,

$$
\omega\left(X_{f}, Y\right)=d f(Y)=Y(f)
$$

Also, we have another important subset of vector field of a symplectic manifold, which is called locally Hamiltonian.

Definition 25. A vector field $X$ of a symplectic manifold $(M, \omega)$ is called locally Hamiltonian if $\mathscr{L}_{X}(\omega)=0$. Since $\omega$ is closed, by the Cartan's magic formula,

$$
\mathscr{L}_{X}(\omega)=i_{X} d \omega+d\left(i_{X} \omega\right)=d\left(i_{X} \omega\right)
$$

Therefore, equivalently, a vector field is locally Hamiltonian if $i_{x} \omega$ is closed.

By the Poincaré lemma [Abraham, Marsden and Ratiu 1993], if $\omega \in \Omega^{k}(M)$ is closed, for every $x \in M$ there is an open neighborhood $U$ of $x$ for which $\left.\omega\right|_{U} \in \Omega^{k}(U)$ is exact. As long as $i_{X} \omega$ is closed, there locally is $h \in C^{\infty}(M)$ such that $i_{X} \omega=d h$. Hence, locally $X=X_{h}$ and therefore it justifies its name.

For every $\omega \in \Omega^{k}(M)$, and every $X \in \mathfrak{X}(M)$

$$
\frac{d}{d t} \varphi_{t}^{X} \omega=\left(\varphi_{t}^{X}\right)^{*}\left(\mathscr{L}_{X}(\omega)\right)
$$

where $\varphi_{t}^{X}$ is the flow of the vector field $X$ [Marsden and Ratiu 2002] . If $X$ is locally Hamiltonian, then

$$
\frac{d}{d t} \varphi_{t}^{X} \omega=0
$$

So the flow $\varphi_{t}^{X}$ is a local symplectomorphism, if, and only if, $X$ is locally Hamiltonian.

Using the Hamiltonian vector field, we can define a Poisson bracket on the smooth functions of $(M, \omega)$.

Proposition 26. The bilinear, skew-symmetric bracket on $C^{\infty}(M)$, defined by,

$$
\{f, g\}:=\omega\left(X_{f}, X_{g}\right)=X_{f}(g)=-X_{g}(f)
$$

is a Poisson Bracket.

The bilinearity and the skew-symmetry are inherited from $\omega$, thus is just necessary to check the Leibniz and Jacobi rules. But, before we prove the above proposition, we will discuss some proprieties of this bracket.

Firstly, we have that the above bracket commutes with the flow of locally Hamiltonian vector fields, as can be seen in the next proposition. 
Proposition 27. If $X$ is a locally Hamiltonian vector field, then

$$
\left(\varphi_{t}^{X}\right)^{*}\{f, g\}=\left\{\left(\varphi_{t}^{X}\right)^{*} f,\left(\varphi_{t}^{X}\right)^{*} g\right\} .
$$

Proof. If $X$ is locally Hamiltonian then $\left(\varphi_{t}^{X}\right)^{*} \omega=\omega$, so

$$
\left(\varphi_{t}^{X}\right)^{*}\{f, g\}(x)=\left(\varphi_{t}^{X}\right)^{*}\left(\omega\left(X_{f}, X_{g}\right)(x)\right)=\omega\left(X_{f}, X_{g}\right)\left(\varphi_{t}^{X}(x)\right)=\left\{\left(\varphi_{t}^{X}\right)^{*} f,\left(\varphi_{t}^{X}\right)^{*} g\right\}
$$

Taking the time derivative of the left-hand side of equation (2.3.1) at $t=0$, one has

$$
\left.\frac{d}{d t}\right|_{t=0}\left(\left(\varphi_{t}^{X}\right)^{*}\{f, g\}\right)=\mathscr{L}_{X_{h}}(\{f, g\}),
$$

while taking the time derivative at $t=0$ of the the right-hand side, one has

$$
\begin{aligned}
\left.\frac{d}{d t}\right|_{t=0}\left(\left\{\left(\varphi_{t}^{X}\right)^{*} f,\left(\varphi_{t}^{X}\right)^{*} g\right\}\right) & =\left\{\left.\frac{d}{d t}\right|_{t=0}\left(\varphi_{t}^{X}\right)^{*} f,\left.\left(\varphi_{t}^{X}\right)^{*} g\right|_{t=0}\right\}+\left\{\left.\left(\varphi_{t}^{X}\right)^{*} f\right|_{t=0},\left.\frac{d}{d t}\right|_{t=0}\left(\varphi_{t}^{X}\right)^{*} g\right\} \\
& =\left\{\mathscr{L}_{X_{h}}(f), g\right\}+\left\{f, \mathscr{L}_{X_{h}}(g)\right\}
\end{aligned}
$$

Therefore, we proved the following corollary.

Corollary 28. For every $f, g, h \in C^{\infty}(M)$ the following identity holds:

$$
\mathscr{L}_{X_{h}}(\{f, g\})=\left\{\mathscr{L}_{X_{h}}(f), g\right\}+\left\{f, \mathscr{L}_{X_{h}}(g)\right\}
$$

Now, we are ready to prove the Jacobi and Leibniz identites:

Proof. - The Jacobi Identity:

$$
\{h,\{f, g\}\}=\mathscr{L}_{X_{h}}(\{f, g\})
$$

thus, by the corollary (28) we have

$$
\begin{aligned}
\{h\{f, g\}\} & =\mathscr{L}_{X_{h}}(\{f, g\})=\left\{\mathscr{L}_{X_{h}}(f), g\right\}+\left\{f, \mathscr{L}_{X_{h}}(g)\right\} \\
& =\{\{h, f\}, g\}+\{f,\{h, g\}\} \\
& =\{g,\{h, f\}\}-\{f,\{g, h\}\} .
\end{aligned}
$$

- Leibniz Rule

$$
\{g, h f\}=X_{g}(h f)=h X_{g}(f)+f X_{g}(h)=h\{g, f\}+f\{g, h\} .
$$


Note that a necessary condition to the bracket of a symplectic manifold to satisfy the Jacob identity is $\mathscr{L}_{X_{h}}(\omega)=d\left(i_{X_{h}} \omega\right)$, and this is true for all vector fields only when $\omega$ is closed. According to the proposition (9) the bracket defined in the proposition (26) has even Rank, and, therefore, it will be the Rank of the symplectic form $\omega$. Since the $\omega$ is non-degenerated, every symplectic manifold must have an even dimension.

In the Section 2.5, we will see that locally, every even dimensional manifold admits a symplectic structure. Although there is no general condition for an arbitrary even-dimensional manifold to admit a symplectic structure, certainly, the possible obstructions have a topological nature. However, in dimension two, there is an answer: a 2-dimensional manifold admits a symplectic structure if, and only if, it is orientable. [Fernandes and Marcut 2015].

The cotangent bundle of every manifold has a special symplectic structure called canonical form.

let

$$
\begin{gathered}
\pi:=T^{*} M \rightarrow M \\
p=(x, \xi) \mapsto x,
\end{gathered}
$$

be the canonical projection, thus, pointwise, we define the tautological 1-form by the following Definition 29. The tautological 1-form on $T^{*} M$ as

$$
\Theta_{p}=\pi_{P}^{*} \xi \in T_{p}^{*}\left(T^{*} M\right)
$$

Let $\left(U_{i}, x_{1}^{i}, \ldots, x_{n}^{i}\right)$ be an atlas to $M$. One can construct the manifold structure, $\left(T^{*} U_{i}, x_{1}, \ldots\right.$, $\left.x_{n}, p_{1}, \ldots, p_{n}\right)$, to $T^{*} M$ using, for a $m \in M$, the basis $\left\{\left(d x_{1}\right)_{m}, \ldots,\left(d x_{n}\right)_{m}\right\}$ of $T_{m}^{*} M$ and the relation $\alpha_{m}=\sum_{i=1}^{n} \xi_{i}\left(d x^{i}\right)_{m}$. Thus, at this coordinates,

$$
\Theta=\sum_{i=1}^{n} p_{i} d x^{i}
$$

From this definition, it is possible to induce a symplectic form on the cotangent bundle of $M$, called canonical symplectic 2 -form $\omega$, as minus the exterior derivative of $\Theta$, i.e.,

$$
\omega=-d \Theta
$$

In the same local coordinates,

$$
\omega=\sum_{i=1}^{n} d x^{i} \wedge d p^{i}
$$

\subsubsection{The Lie-Poisson structure on $\mathfrak{g}^{*}$}

A linear Poisson structure on a vector space $V$, is defined by the property that the Poisson bracket of two linear functions is again a linear function. The linear Poisson structures are closely related to Lie algebras structures on the dual space of $V$. 
Let a finite-dimensional Lie algebra, $\mathfrak{g}$, with bracket $[\cdot, \cdot]$, we denote the dual space of $\mathfrak{g}$ by $\mathfrak{g}^{*}$. Every element $e$ of $\mathfrak{g}$ defines a linear functional $e^{*}$ by

$$
\begin{aligned}
e^{*}: \mathfrak{g}^{*} & \rightarrow \mathbb{R} \\
\xi & \mapsto\langle\xi, e\rangle:=\xi(e) .
\end{aligned}
$$

If $f \in C^{\infty}\left(\mathfrak{g}^{*}\right)$, then $d_{\xi} f: T_{\xi} \mathfrak{g}^{*} \rightarrow \mathbb{R}$ and $T_{\xi} \mathfrak{g}^{*}$ is naturally isomorphic with $\mathfrak{g}^{*}$, one can think of $d_{\xi} f$ as an element of $\left(\mathfrak{g}^{*}\right)^{*} \simeq \mathfrak{g}$. Thus, one can define $\{\cdot, \cdot\}: C^{\infty}\left(\mathfrak{g}^{*}\right) \times C^{\infty}\left(\mathfrak{g}^{*}\right) \rightarrow C^{\infty}\left(\mathfrak{g}^{*}\right)$ by

$$
\{f, g\}(\xi):=\left\langle\xi,\left[d_{\xi} f, d_{\xi} g\right]\right\rangle
$$

If $\left(e_{1}, \ldots, e_{d}\right)$ is a basis of $\mathfrak{g}$, and $\left(x_{1}, \ldots, x_{d}\right)$ is the dual basis one can define $\left(x_{1}, \ldots, x_{n}\right)$ global coordinates on $\mathfrak{g}$ by $x_{i}(v)=\left\langle e_{i}^{*}, v\right\rangle$ and global coordinates on $\mathfrak{g}^{*}$ by $y_{i}(\alpha)=\left\langle e_{i}, \alpha\right\rangle$.

If $f \in C^{\infty}\left(\mathfrak{g}^{*}\right)$

$$
d_{\xi} f=\left.\frac{d}{d t}\right|_{t=0} f(\xi+\alpha t)=\sum_{i=1}^{n} \frac{\partial f}{\partial y_{i}}(\xi) \alpha_{i}=\left\langle\sum_{i=1}^{n} \frac{\partial f}{\partial y_{i}}(\xi), \alpha\right\rangle
$$

and

$$
\begin{aligned}
{\left[d_{\xi} f, d_{\xi} g\right] } & =\sum_{i, j} \frac{\partial f}{\partial y_{i}}(\xi) \frac{\partial g}{\partial y_{j}}(\xi)\left[e_{i}, e_{j}\right]=\sum_{i, j} \frac{\partial f}{\partial y_{i}}(\xi) \frac{\partial g}{\partial y_{j}}(\xi) \sum_{k} c_{i, j}^{k} e_{k} \\
& =\sum_{i, j}\left(\sum_{k} c_{i, j}^{k} y_{k}\right) \frac{\partial f}{\partial y_{i}}(\xi) \frac{\partial g}{\partial y_{j}}(\xi)
\end{aligned}
$$

By the bilinearity of $\{\cdot, \cdot\}$ and $[\cdot, \cdot]$

$$
\left\{f^{*}, g^{*}\right\}=[g, f]^{*}, \forall f, g \in \mathfrak{g},
$$

and also

$$
\left\{\left\{x^{i}, x^{j}\right\}, x^{k}\right\}=\left[\left[e_{i}, e_{j}\right], e_{k}\right]^{*}
$$

and this proves the Jacobi identity.

On [Laurent-Gengoux, Pichereau and Vanhaecke 2012], one finds the following result Theorem 30. For every finite-dimensional vector space $V$, there is a natural one-to-one conrrespondence between a linear Poisson structures on $V^{*}$ and Lie algebra structures on $V$.

\subsubsection{The tangent lifts of a Poisson structure}

Given a Poisson manifold $(M, \Pi)$ there is a canonical way to define a Poisson structure, $\Pi_{T M}$, on the tangent bundle $T M$. First of all, we define the tangent lift of a smooth function on $M$. 
Definition 31. Let $f \in C^{\infty}(M)$, we call the the tangent lift of $f$, the function $\bar{f} \in C^{\infty}(T M)$ defined as follow: for every $v \in T M$

$$
\bar{f}(v)=v(f)
$$

using a analogous construction of the cotangent coordinates, one can construct tangent coordinates $\left(x^{i}, y^{i}\right)$ associated to the bases $\left\{\left(\frac{\partial}{\partial x^{1}}\right)_{x}, \ldots,\left(\frac{\partial}{\partial x^{n}}\right)_{x}\right\}$ of $T^{*} M$. Thus, in this coordinates,

$$
\bar{f}=\sum_{i=1}^{n} \frac{\partial f}{\partial x^{i}} y^{i}
$$

Those functions are called fiber-wise linear function. Fiber-wise linear in the sense that, for every fiber $\operatorname{proj}^{-1}(x)$ on $T M$, the function $\bar{f}$ is a linear function.

We define the Poisson structure, $\Pi_{T M}$, on $T M$ through the next theorem

Theorem 32. Let $(M, \Pi)$ be a Poisson manifold. The Poisson bivector field $\Pi_{T M}$ defined locally by

$$
\Pi_{T M}=\sum_{i, j} \Pi^{i j}(x) \frac{\partial}{\partial x^{i}} \wedge \frac{\partial}{\partial y^{j}}+\frac{1}{2} \sum_{i, j, k} \frac{\partial \Pi^{i j}}{\partial x^{k}} y^{k} \frac{\partial}{\partial y^{i}} \wedge \frac{\partial}{\partial y^{j}},
$$

is the unique Poisson structure such that the associated bracket satisfies

$$
\{\bar{f}, \bar{g}\}_{T M}=\{f, g\}, \text { for all } f, g \in C^{\infty}(M) .
$$

The Poisson structure defined on the above theorem is called fiber-wise linear Poisson structure on $T M$.

\subsection{The Poisson category}

The Poisson category is the category which objects are Poisson manifolds and which morphisms are defined here bellow.

Definition 33. Let $\left(M,\{\cdot, \cdot\}_{M}\right)$ and $\left(N,\{\cdot, \cdot\}_{N}\right)$ be two Poisson manifolds. A Poisson map, or a Poisson morphism, is a smooth map $\psi: M \rightarrow N$, satisfying:

$$
\{f \circ \psi, g \circ \psi\}_{M}=\{f, g\}_{N} \circ \psi
$$

for all $f, g \in C^{\infty}(N)$. Or, equivalently, for the Poisson bivector

$$
\psi_{*}\left(\Pi_{M}\right)_{x}=\left(\Pi_{N}\right)_{x}
$$

where $\Pi_{M}$ and $\Pi_{N}$ are the Poisson bivector associated to $\{\cdot, \cdot\}_{M}$ and $\{\cdot, \cdot\}_{N}$, respectively.

The following proposition ensures that we do have a category. 
Proposition 34. Let $\left(M, \Pi_{M}\right),\left(N, \Pi_{N}\right)$ and $\left(P, \Pi_{P}\right)$ be Poisson manifolds, and let $\psi: M \rightarrow N$ and $\varphi: N \rightarrow P$ be differentiable maps.

I. If $\psi$ and $\varphi$ are Poisson maps, then $\varphi \circ \psi$ is a Poisson map,

II. If $\psi$ and $\varphi \circ \psi$ are Poisson maps and, furthermore, if $\psi$ is surjective, then $\psi$ is a Poisson morphism. In particular, if $\psi$ is a Poisson morphism and a diffeomorphism, then $\psi^{-1}$ is a Poisson map. In this case, we say that $\psi$ is a Poisson diffeomorphism, or an isomorphism on the category of Poisson manifolds.

Proof. The first claim comes immediately from the definition of the Poisson map. For the second one, let $x \in M$, then

$$
\begin{aligned}
\left(\#_{P}\right)_{\varphi(\psi(X))} & =T_{x}(\varphi \circ \psi) \circ\left(\#_{M}\right)_{x} \circ T_{x}^{*}(\varphi \circ \psi) \\
& =T_{\psi(x)}(\varphi) \circ T_{x}(\psi) \circ\left(\#_{M}\right)_{x} \circ T_{x}^{*}(\psi) \circ T_{\psi(x)}^{*}(\varphi) \\
& =T_{\psi(x)}(\varphi) \circ\left(\#_{N}\right)_{\psi(x)} \circ T_{\psi(x)}^{*}(\varphi) .
\end{aligned}
$$

The Poisson vector fields are invariant under Poisson morphism.

Proposition 35. Let $\psi: M \rightarrow N$ be a Poisson map between $\left(M,\{\cdot, \cdot\}_{M}\right)$ and $\left(N,\{\cdot, \cdot\}_{N}\right)$. For every $h \in C^{\infty} N$

$$
X_{h}=T \psi\left(X_{h \circ \psi}\right)
$$

or, equivalently,

$$
\left(\#_{N}\right)_{(\psi(x))}=T_{x} \psi \circ\left(\#_{M}\right)_{x} \circ T_{x}^{*} \psi, \quad \forall x \in M .
$$

Proof. Using the definitions of Hamiltonian vector field and Poisson map, for every $f \in C^{\infty} N$, we have

$$
\begin{aligned}
X_{h \circ \psi}(f \circ \psi) & =\{h \circ \psi, f \circ \psi\}_{M}=\{h, f\}_{N} \circ \psi \\
& =X_{h}(f) \circ \psi .
\end{aligned}
$$

One can understand equivalent equation by the commutativity of the following diagram, and, to prove it is necessary to do nothing but replace the Poisson bracket by the bivector field.

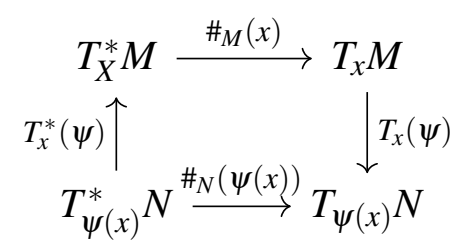


Furthermore for every $h \in C^{\infty} M, \mathscr{L}_{X_{h}}(\Pi)=0$. So, the flow $\varphi_{X_{h}}$ is a local Poisson diffeomorphisms and if $X_{h}$ is a complete vector field, $\varphi_{X_{h}}$ is a global Poisson diffeomorphisms.

Example 36. Let $M=\mathbb{R}^{2 n}$ with coordinates $\left(p^{1}, \ldots, p^{n}, q^{1}, \ldots, q^{n}\right)$ and the Poisson bivector field given by

$$
\Pi=\sum_{i=1}^{n} \frac{\partial}{\partial q^{i}} \wedge \frac{\partial}{\partial p^{i}},
$$

let $N=\mathbb{R}^{n}$ with coordinates $\left(q^{1}, \ldots, q^{n}\right)$ and the trivial bracket. The projection proj: $\mathbb{R}^{2 n} \rightarrow \mathbb{R}^{n}$, $\left(p^{1}, \ldots, p^{n}, q^{1}, \ldots, q^{n}\right) \mapsto\left(p^{1}, \ldots, p^{n}\right)$ is a Poisson map.

Example 37. If there is morphism between two Lie algebras, $\varphi: \mathfrak{g} \rightarrow \mathfrak{h}$, the dual transformation, $\varphi^{*}: \mathfrak{h}^{*} \rightarrow \mathfrak{g}^{*}$, is a Poisson morphism in the opposite direction. Indeed, for each $f, g \in C^{\infty}\left(\mathfrak{g}^{*}\right)$ and $\xi \in h^{*}$, we have

$$
\begin{aligned}
\left\{f \circ \varphi^{*}, g \circ \varphi^{*}\right\}_{\mathfrak{h}^{*}}(\xi) & =\left\langle\xi,\left[d_{\xi}\left(f \circ \varphi^{*}\right), d_{\xi}\left(g \circ \varphi^{)}\right]\right\rangle\right. \\
& =\left\langle\xi,\left[d_{\varphi^{*}(\xi)} f \circ d_{\xi} \varphi^{*}, d_{\varphi^{*}(\xi)} g \circ d_{\xi} \varphi^{*}\right]\right\rangle \\
& =\left\langle\xi,\left[d_{\varphi^{*}(\xi)} f \circ \varphi^{*}, d_{\varphi^{*}(\xi)} g \circ \varphi^{*}\right]\right\rangle \\
& =\left\langle\xi,\left[\varphi\left(d_{\varphi^{*}(\xi)} f\right), \varphi\left(d_{\varphi^{*}(\xi)} g\right)\right]\right\rangle \\
& =\left\langle\xi, \varphi\left(\left[d_{\varphi^{*}(\xi)} f, d_{\varphi^{*}(\xi)} g\right]\right)\right\rangle \\
& =\left\langle\xi,\left[d_{\varphi^{*}(\xi)} f, d_{\varphi^{*}(\xi)} g\right] \circ \varphi^{*}\right\rangle \\
& =\left\langle\varphi^{*}(\xi),\left[d_{\varphi^{*}(\xi)} f, d_{\varphi^{*}(\xi)} g\right]\right\rangle \\
& =\{f, g\}_{\mathfrak{g}^{*}}\left(\varphi^{*}(\xi)\right) .
\end{aligned}
$$

Since "take the dual" is functorial, so is the above definition. In this way, we have a contravariant functor from the category of Lie algebras to the category of Poisson manifolds.

Definition 38. A Poisson submanifold is a submanifold $N$ of a Poisson manifold $M$ such that, $N$ has a Poisson structure that makes the inclusion map a Poisson map.

Lemma 39. $N \subset M$ is a Poisson submanifold if, and only if, each tangent space $T_{x} N$ contains the image of $\#_{x}: T_{x}^{*} M \rightarrow T_{x} M$, in other words, if, and only if, all Hamiltonian vector fields are tangent to $\mathrm{N}$.

\subsection{The splitting theorem for Poisson manifolds}

A classic result of symplectic geometry is the Darboux's theorem [Dufour and Zung 2006]. Roughly speaking, it says that two symplectic manifold of the same dimension locally look like the same. Formally 
Theorem 40 (Darboux). Let $\omega$ be a non-degenerate two-form on a manifold $M$. Then $d \omega=0$ if, and only if, for every $m \in M$, there exists a chart $\left(U, q^{1}, \ldots, q^{n}, p^{1}, \ldots, p^{n}\right)$ such that $q^{i}(m)=$ $p^{i}(m)=0$ and

$$
\omega=\sum_{i=1}^{n} d q^{i} \wedge d p^{i}
$$

The difference between a generic Poisson structure and one that comes from a symplectic form is that the Rank of the generic one does not need to be maximal. In these terms, it is impossible that all the Poisson structures are locally the same. However, we have an analogous result, the Splitting Theorem, which tells us that for a Poisson manifold, at any point $x \in M$, the Poisson structure splits in a non-degenerate part, the symplectic one, and in a new Poisson structure that vanishes at $x$. The Darboux's theorem is just a corollary of the Splitting theorem. To prove it, we will need the following result [Laurent-Gengoux, Pichereau and Vanhaecke 2012]:

Theorem 41 (Straightening theorem). Let $M$ be a smooth manifold, $X \in \mathfrak{X}(M)$ and $x \in M$ satisfying $X(x) \neq 0$. Then, there is a coordinate neighborhood $\left(U, y_{1}, \ldots y_{m}\right)$ such that $\left.X\right|_{U}=\frac{\partial}{\partial y_{1}}$. Theorem 42 (Splitting theorem). Let $(M, \Pi)$ be a Poisson manifold, let $x \in M$ and let $2 r$ be the Rank of $\Pi$ at $x$. There exists a coordinate neighborhood $\left(U, q^{1}, \ldots, q^{r}, p^{1}, \ldots, p^{n}, z^{1}, \ldots, z^{s}\right)$, centered at $x$, such that, on $U$,

$$
\Pi=\sum_{i=1}^{r} \frac{\partial}{\partial q^{i}} \wedge \frac{\partial}{\partial p^{i}}+\sum_{1 \leq k<l \leq s} \Pi^{k, l} \frac{\partial}{\partial z^{k}} \wedge \frac{\partial}{\partial z^{l}} .
$$

The functions $\Pi^{k, l}$ are smooth functions which depend only on $z=\left(z_{1}, . ., z_{s}\right)$, and which vanish at $z=0$. The local coordinates $\left(q_{1}, \ldots, q_{r}, p_{1}, \ldots, p_{r}, z_{1}, \ldots, z_{s}\right)$ are called splitting coordinates centered at $x$.

In [Weinstein 1983], Alan Weinstein presents the following demonstration.

Proof. Since this result is local, we can prove it considering $M=\mathbb{R}^{n}$. We will proceed by induction on $r$. If $r=0$, we are done. The induction hypotheses are that the above theorem is true for every Poisson manifold, at every point where the Rank is $2(r-1)$. Take $x$ in a Poisson manifold $(M, \Pi)$ such that $\operatorname{Rank}_{x} \Pi=2 r$.

Since $\operatorname{Rank}_{x} \Pi>0$, there exists a local function $p$ which Hamiltonian vector field \# $(d p)=$ $X_{p}$ does not vanish at $x$. Without losing generality, we may suppose that $p(x)=0$. Since $X_{p}(x) \neq 0$, there exists, by the straightening theorem, a system of coordinates $\left(q, y_{2}^{\prime}, \ldots, y_{d}^{\prime}\right)$ on a neighborhood $U^{\prime}$ centered at $x$, such that $X_{p}=\frac{\partial}{\partial q}$. For $i=2, \ldots, d$, we have that

$$
\{p, q\}=X_{p}(q)=\frac{\partial q}{\partial q}=1,\left[X_{p}, X_{q}\right]=X_{\{p, q\}}=X_{1}=0, X_{p}\left(y_{i}^{\prime}\right)=0 .
$$

Thus, in terms of these coordinates,

$$
X_{q}=\sum_{i=2}^{d} \alpha_{i} \frac{\partial}{\partial y_{i}^{\prime}}
$$


and the coefficients $\alpha_{i}$ are independent of $q$. Indeed, for any smooth function $f$ we have

$$
\begin{aligned}
& X_{q}\left(X_{p}(f)\right)=X_{p}\left(X_{q}(f)\right) \\
& \Longrightarrow \alpha_{i} \frac{\partial^{2} f}{\partial y_{i} \partial_{q}}=\frac{\partial \alpha_{i}}{\partial q} \frac{\partial f}{\partial y_{i}}+\alpha_{i} \frac{\partial^{2} f}{\partial y_{i} \partial_{q}}
\end{aligned}
$$

So $X_{q}$ is independent of $q$ and does not vanish at $x$. Applying the straightening theorem once more, on the set $\left(q_{0}, y_{2}^{\prime}, \ldots, y_{d}^{\prime}\right)$, we may introduce a system of coordinates $\left(q, p^{\prime}, y_{3}, \ldots, y_{d}\right)$ on a neighborhood of $\mathrm{x}$, where $\left(p^{\prime}, y_{3}, \ldots, y_{d}\right)$ depend only on $\left(y_{2}^{\prime}, \ldots, y_{d}^{\prime}\right)$, with

$$
\frac{\partial}{\partial p^{\prime}}=X_{q}
$$

Substituting $p^{\prime}$ by $p$ we consider $\left(q, p, y_{3}, \ldots, y_{d}\right)$, so the Poisson brackets, in these new coordinates, take the following form,

$$
\begin{aligned}
& \{p, q\}=1 \\
& \left\{q, y_{i}\right\}=\frac{\partial y_{i}}{\partial p^{\prime}}=0 \\
& \left\{p, y_{i}\right\}=\frac{\partial y_{i}}{\partial q}=0
\end{aligned}
$$

and we conclude that the Poisson structure $\Pi$ is given by

$$
\Pi=\frac{\partial}{\partial q} \wedge \frac{\partial}{\partial p}+\sum_{3 \leq k<l<d}\left\{y_{k}, y_{l}\right\} \frac{\partial}{\partial y_{k}} \wedge \frac{\partial}{\partial y_{l}} .
$$

Since $\left\{\left\{y_{k}, y_{l}\right\}, p\right\}=\left\{\left\{y_{k}, y_{l}\right\}, q\right\}=0$ for all $k, l$, we have that $\left\{y_{k}, y_{l}\right\}$ does not depend of $p$ and $q$.

Definition 43. The first term of the above decomposition is called the symplectic part while the second term is called the singular part.

Thus, we can see the Poisson manifold locally as a product $S \times N$ such that $S$ is symplectic and $N$ is Poisson with $\operatorname{Rank}\left(x_{0}\right)=0$. When the Poisson structure is also symplectic, that is, it is non-degenerate, we recover the Darboux theorem.

Now, one can reduce the problem of local classification of Poisson structures to classify the Poisson structures $\Pi$ on $\mathbb{R}^{n}$, such that $\Pi(0)=0$, i.e., we can assume that, locally, every Poisson manifold is $\mathbb{R}^{n}$ and that $\Pi(0)=0$.

\subsection{Symplectic foliation}

The Weinstein's splitting theorem describes the local structure of a Poisson manifold. Globally, roughly speaking, we can think of a Poisson manifold as a disjoint union of symplectic ones, not necessarily of the same dimension. 
We begin this section by presenting some definitions and results on the distribution theory - in differential geometry sense. Next, we discuss the integrability of the distribution generated by the Hamiltonian vector fields and how it gives a symplectic structure on each leaf. We conclude presenting the example of the symplectic leaves of a linear Poisson structure. We follow closely [Vaisman 2012, Dufour and Zung 2006].

\subsubsection{Singular foliations and singular distributions}

A intuitive way to think of a foliation is as a partition of a manifold $M$ in immersed and connected submanifolds, called the leaves of the foliation. We started by defining a regular foliation.

Definition 44. [Moerdijk and Mrcun 2003] Let $M$ be a smooth manifold of dimension $n$. A foliation atlas of co-dimension $q$ on $M$ is an atlas

$$
\left(\varphi_{i}: U_{i} \rightarrow \mathbb{R}^{n}=\mathbb{R}^{n-q} \times \mathbb{R}^{q}\right)_{i \in I}
$$

of $M$ for which the change of coordinates, $\varphi_{i j}$, are locally of the form

$$
\varphi_{i j}=\left(g_{i j}(x, Y), h_{i j}(y)\right)
$$

with respect to the decomposition $\mathbb{R}^{n}=\mathbb{R}^{n-q} \times \mathbb{R}^{q}$. A foliation of co-dimension $\mathbf{q}$ on $M$ is a maximal foliation atlas of $M$.

The above definition includes just the case where the leaves have the same dimension. It is possible to extend this definition to permit leaves of different dimension.

Definition 45. A smooth singular foliation on a smooth manifold $M$, in the sense of StefanSussmann is a partition $\mathscr{F}=\{\mathscr{F} \alpha\}$ of $M$ into a disjoint union of smooth immersed connected submanifolds $\mathscr{F}_{\alpha}$, which satisfies two conditions. For every $x \in M$, let $\mathscr{F}_{x}$ be the leaf that contains $x$ and suppose that its dimension is $d$.

I. There is a smooth local chart of $M$ with coordinates $\left(x_{1}, \ldots, x_{m}\right)$ in a neighborhood $U$ of $x, U=\left\{-\varepsilon<x_{1}<\varepsilon, \ldots,-\varepsilon<x_{m}<\varepsilon,\right\}$, such that the $d$-dimensional disk $\left\{y_{d+1}=\ldots=\right.$ $\left.y_{m}=0\right\}$ coincides with the path-connected component of the intersection of $\mathscr{F}_{x}$ with $U$ which contains $\mathrm{x}$;

II. Each $d$-dimensional disk $\left\{y_{d+1}=c_{d+1}, \ldots, y_{m}=c_{m}\right\}$, where $c_{d+1}, \ldots, c_{m}$ are constants, is entirely contained in some leaf $\mathscr{F} \alpha$.

It is possible to replace the previous definition using sub-bundles of $T M$. A general distribution on $M$ is a choice for every $x \in M$ of subspace $D_{x} \subset T_{x} M$ which dimension may depend on $x$. A general distribution is called smooth if, for any point $x \in M$, there exists a set 
$\left\{X_{1}, \ldots, X_{m}\right\}$ of vector field on $M$ such that $D_{x}=\operatorname{Span}\left\{X_{1}(x), \ldots, X_{m}(x)\right\}$. Of course, $m$ may depend on the point $x$. When the dimension of $D_{x}$ is constant, the distribution is called regular, otherwise, it is called singular.

Every family $C$ of smooth vector fields on $M$ gives rise to a smooth singular distribution $D^{C}$ defined as follows: for each $x \in M, D_{x}^{C}$ is the vector space spanned by the values at $x$ of the vector fields of $C$.

A distribution $D$ is called invariant with respect to a family of smooth vector fields $C$ if, for every $X \in C$, we have

$$
\left(\varphi_{X}^{t}\right)_{*} D_{x}=D_{\varphi_{X}^{t}(x)},
$$

where $\left(\varphi_{X}^{t}\right)$ is the local flow of $X$.

Definition 46. We say that a smooth singular distribution $D$ is integrable if every point of $M$ is contained in a maximal immersed submanifold $W$, such that for every $y \in W$ the tangent space $T_{y} W=D_{y}$.

One can ask about the conditions to guarantee that a distribution is integrable. In the regular case, the answer to this question is given by Frobenius theorem [Warner 1983].

Theorem 47 (Frobenius). The condition to integrability on the regular case is that the distribution is involutive, i. e., if $[X, Y] \in D$ whenever $X$ and $Y$ are smooth vector fields lying in $D$.

For the singular case, the answer is provided by the Stefan-Sussmann's theorem [Dufour and Zung 2006].

Theorem 48 (Stefan-Sussmann). Let $D$ be a smooth singular distribution on $M$. Then the following three conditions are equivalent:

- $D$ is integrable.

- $D$ is generated by a family $C$ of smooth vector fields, and it is invariant with respect to $C$.

- $D$ is the tangent distribution of a smooth singular foliation $\mathscr{F}$.

The classical notion of involutivity can be extended to general distributions as follows.

Definition 49. A distribution $D$ is said involutive if there exists a Lie subalgebra $\mathfrak{X}(D)$ of $\mathfrak{X}(M)$ such that the span of the values at $x$ of the fields of $\mathfrak{X}(D)$ equals $D_{x}$, for all $x \in M$.

The following theorem [Vaisman 2012] gives a condition for integrability of general smooth distribution in terms of involutivety.

Theorem 50 (Viflyantsev-Frobenius). A smooth distribution $D$ is integrable if, and only if, it satisfies the following two conditions: 
I. $D$ is involutive;

II. For all $x \in M$ and for all $v \in D_{x}$, there is a path $\gamma(t)$, such that $\gamma(0)=x, \gamma^{\prime}(0)=v$ and which $\operatorname{Rank}\left(D_{\gamma(t)}\right)$ is constant.

\subsubsection{The symplectic foliation of a Poisson manifold}

Using the characteristic spaces introduced on definition (8), one can define a differentiable singular distribution on every Poisson manifold $(M, \Pi)$ as follows:

Definition 51. For all $x \in M$,

$$
C_{x}=\operatorname{Span}\left\{\Pi_{x}(d f) \mid f \in C^{\infty}(M)\right.
$$

is called the characteristic distribution.

By definition, $C$ is generated by the family of Hamiltonian vector fields and it is invariant with respect to them. Furthermore, by the relation

$$
X_{\{f, g\}}=\left[X_{f}, X_{g}\right]
$$

the set of Hamiltonian vector fields is a Lie algebra and $C$ is an involutive distribution.

In the case of regular Poisson structure, the integrability of the characteristic distribution comes from the Frobenius' theorem. Otherwise, since the flow of every Hamiltonian vector field is a Poisson automorphism, one can conclude the integrability of the characteristic distribution using Theorem (48) or (50). We will denote the correspondent singular foliation by $\mathscr{F}$.

Since for all $x \in M$, the dimension of $D_{x}$ is even, the leaves of $\mathscr{F}$ are even dimension submanifolds.

For every $x \in M$, one defines a symplectic structure on $\mathscr{F}_{x}$ as follows. If $f, g \in C^{\infty}\left(\mathscr{F}_{x}\right)$, we can extend them locally to functions $\tilde{f}, \tilde{g} \in C^{\infty}(M)$, we define the bracket between $f$ and $g$ as

$$
\{\tilde{f}, \tilde{g}\}(x)=\{f, g\}=X_{f}(g)(x)=-X_{g}(f)(x) .
$$

Since the bracket is computed along the flow of $X_{f}$ and $X_{g}$, which belongs to $\mathscr{F}_{x}$, the result depends only $\tilde{f}$ and $\tilde{g}$. Now, we can call this foliation by symplectic foliation of the Poisson manifold $M$.

There is a more explicit construction to the symplectic foliation, which can be seen at [Laurent-Gengoux, Pichereau and Vanhaecke 2012]. In this way, the idea is to construct the manifold using the Hamiltonian vector field. For each $x \in M$, we define the subset of $M$

$$
\mathscr{P}_{x}(M):=\left\{x^{\prime} \in M \mid \exists \text { piecewise Hamiltonian path in } M \text { from } x \text { to } x^{\prime}\right\}
$$

Note that the subsets $\mathscr{P}_{x}(M)$ define a partition of $M$. 
We choose splitting coordinates $\varphi=\left(q_{1}, \ldots, q_{r}, p_{1}, \ldots, p_{r}, z_{1}, \ldots, z_{s}\right)$, on a neighborhood $U$ of $x$, and we define

$$
\mathscr{P}_{x}(U, \varphi):=\left\{x^{\prime} \in U \mid z_{1}\left(x^{\prime}\right)=\ldots=z_{s}\left(x^{\prime}\right)=0\right\}
$$

Thus, $\mathscr{P}_{x}(U, \varphi)$ is an embedded submanifold of $M$ of dimension $2 r,\left(q_{1}, \ldots, q_{r}, p_{1}, \ldots, p_{r}\right)$ forms a system of coordinates on $\mathscr{P}_{x}(U, \varphi)$ and we have

$$
\Pi_{\mathscr{P}}=\sum_{i=1}^{r}=\frac{\partial}{\partial q_{i}} \wedge \frac{\partial}{\partial p_{i}} .
$$

The map $i:\left(\mathscr{P}_{x}(U, \varphi), \Pi_{\mathscr{P}}\right) \rightarrow(M, \Pi)$ is a Poisson map. Indeed, take two functions $f, g \in$ $C^{\infty}(U)$, for every $x^{\prime} \in \mathscr{P}_{x}(U, \varphi)$, we have that

$$
\begin{aligned}
\{f, g\}\left(i\left(x^{\prime}\right)\right) & =\{f, g\} x^{\prime}=\sum_{i=1}^{r}\left(\frac{\partial f}{\partial q_{i}}\left(x^{\prime}\right) \frac{\partial g}{\partial p_{i}}\left(x^{\prime}\right)-\frac{\partial g}{\partial q_{I}}\left(x^{\prime}\right) \frac{\partial f}{\partial p_{i}}\left(x^{\prime}\right)\right) \\
& =\{f \circ i, g \circ i\}_{\mathscr{P}}\left(x^{\prime}\right) .
\end{aligned}
$$

Proposition 52. Let $x$ be a point in a Poisson manifold $(M, \Pi)$ and let $\varphi=\left(q_{1}, \ldots, q_{r}, p_{1}, \ldots, p_{r}\right.$, $\left.z_{1}, \ldots, z_{s}\right)$ be the splitting coordinates at $x$, on a coordinate neighborhood $U$, with $\varphi(U)$ convex. Then $\mathscr{P}_{x}(U)=\mathscr{P}_{x}(U, \varphi)$.

Proof. For $f \in C^{\infty}(U)$ and $x^{\prime} \in \mathscr{P}(U, \varphi)$, we have that $\xi_{f}\left(z_{i}\right)\left(x^{\prime}\right)=\sum_{l=1}^{s}\left\{z_{i}, z_{l}\right\}\left(x^{\prime}\right) \frac{\partial F}{\partial z_{l}}=0$ since $\left\{z-i, z_{l}\right\}\left(x^{\prime}\right)=0$. It follows that Hamiltonian vector fields of $U$ are tangent to $\mathscr{P}_{x}(U, \varphi)$ at points $x^{\prime} \in \mathscr{P}_{x}(U, \varphi)$. Thus, a piecewise Hamiltonian path in $U$, which starts at $x$, must stay in $\mathscr{P}_{x}(U, \varphi)$, so $\mathscr{P}_{x}(U) \subset \mathscr{P}_{x}(U, \varphi)$. Now, take $x^{\prime} \in \mathscr{P}$, consider the linear function $h$ on $U$, given by

$$
h:=\sum_{i=1}^{r}\left(q_{i}\left(x^{\prime}\right) p_{i}-p_{i}\left(x^{\prime}\right) q_{i}\right),
$$

the integral curve of $\xi_{h}$ is given by

$$
\begin{gathered}
\dot{q}_{i}=\frac{\partial h}{\partial p_{i}}=q_{1}\left(x^{\prime}\right) \\
\dot{p}_{i}=\frac{\partial h}{\partial q_{i}}=p_{i}\left(x^{\prime}\right) \\
\gamma(t)=\left(q_{1}\left(x^{\prime}\right) t, \ldots, q_{r}\left(x^{\prime}\right) t, p_{1}\left(x^{\prime}\right) t, \ldots, p_{r}\left(x^{\prime}\right) t\right) .
\end{gathered}
$$

Since $U$ is convex, $\gamma \subset U$. Thus, $x^{\prime} \in \mathscr{P}_{x}(U)$.

We build a topology and a differential structure on each one of the subsets $\mathscr{P}_{x}$. A subset $V \subset \mathscr{P}_{x}$ is, by definition, an open subset if, and only if, for all splitting coordinates $(U, \varphi)$ at $x \in V$, with $\varphi(U)$ convex, $V \cap U$ is an open subset of $\mathscr{P}_{x}(U)=\mathscr{P}_{x}(U, \varphi)$. Similarly, an algebra of functions is defined on $\mathscr{P}_{x}(U)$ by saying that $f: \mathscr{P}_{x}(M) \rightarrow \mathbb{R}$ is smooth, when its restriction to every such $\mathscr{P}_{x}(U)=\mathscr{P}_{x}(U, \varphi)$ is smooth. Since the topology and the differential structure on 
these subsets are intrinsically defined, as embedded submanifolds of $M$, these definitions yield a well-defined manifold structure on $\mathscr{P}_{x}(M)$.

On dimension two, the symplectic foliation is quite simple. Basic there are two kinds of leaves: the zero and two-dimensional leaves. The 0-dimensional leaves are the zeros of $\Pi$. The 2-dimensional leaves are the connected component of the set $\left\{x \in M \mid \Pi_{x} \neq 0\right\}$.

The corollary (6) implies that every Casimir functions are constant along with the symplectic leaves.

Proposition 53. Let $M$ be a Poisson manifold, $S \subset M$ is a symplectic leaf, and $f$ is a Casimir function, then $f$ is constant on $S$.

Let $M$ be a manifold and $S(M)$ a general foliation such that

- every leaf $S$ of $S(M)$ is endowed with a symplectic structure $W_{S}$;

- if $f \in C^{\infty}(M)$, the vector field $X_{f}$ defined by the Hamiltonian vector field of $\left.f\right|_{S(x)}$, i. e.,

$$
X_{f}(x)=\left.X_{f}\right|_{S(x)},
$$

is smooth.

Them we define

$$
\{f, g\}=X_{f}(g),
$$

for all $F, G \in C^{\infty}(M)$. So the Poisson structure can be defined by its symplectic foliation.

\subsubsection{The symplectic foliation of a linear Poisson structure}

To construct a nontrivial example, we returned to the linear Poisson structures. The goal of this section is to show the following proposition.

Proposition 54. The symplectic leaves of the Lie-Poisson structure of $\mathfrak{g}^{*}$ are the orbits of the coadjoint representation of any connected Lie group $G$ whose Lie algebra is $\mathfrak{g}$.

We follow the demonstration presented in [Vaisman 2012], but readers are also referred to [Dufour and Zung 2006, Laurent-Gengoux, Pichereau and Vanhaecke 2012] for further information. In a coordinate system $x_{i}$, the equation (2.3.6) implies that one can describe the set of Hamiltonian vector fields of a Poisson linear structure by: let $f \in C^{\infty}\left(\mathfrak{g}^{*}\right)$, so

$$
X_{f}=\sum_{i} \frac{\partial f}{\partial x_{i}} X_{x_{i}} .
$$

The previous equation shows that the characteristic distribution of a Lie-Poisson structure is generated by the set of Hamiltonian vector fields, $\mathfrak{X}_{\text {lin }}$, of the linear functions on $\mathfrak{g}^{*}$. Together, 
the equations (2.3.6) and (2.3.8) define a Lie algebra morphism $\mathfrak{g} \rightarrow \mathfrak{X}_{\text {lin }}$ which the kernel is the center of $\mathfrak{g}$.

Supposed that $G$ is a Lie group that $\mathfrak{g}$ is isomorphic to $\operatorname{Lie}(G)$. We define a representation of $G$ on $G$ by

$$
\begin{aligned}
A: G & \rightarrow A u t(G) \\
h & \mapsto A_{h}(g)=h g h^{-1}
\end{aligned}
$$

Through the differential of $A$ on the identity element, $e$, we can define a representation of $G$ on $\mathfrak{g}$

$$
\begin{aligned}
A d: G & \rightarrow A u t(\mathfrak{g}) \\
h & \mapsto A(h)_{*}(X)
\end{aligned}
$$

and we define the coadjoint representation by

$$
\left\langle A d_{g}^{*}(\xi), x\right\rangle=\left\langle\xi, A d_{g^{-1}}(x)\right\rangle
$$

The tangent vectors to coadjoint orbits can be describe by the following: let $x \in \mathfrak{g}$, let $\mathscr{O}$ be an orbit of coadjoint action and $\mu \in \mathscr{O}$. It is possible to define a curve on $\mathscr{O}$ as

$$
\mu(t)=A d_{e^{-t x}}^{*}(\mu)
$$

And we have in $y \in \mathfrak{g}$

$$
\begin{aligned}
\left\langle\mu^{\prime}(0), y\right\rangle & =\left\langle\left.\frac{d}{d t}\right|_{t=0} \mu(t), y\right\rangle=\left.\frac{d}{d t}\right|_{t=0}\langle\mu(t), y\rangle=\left.\frac{d}{d t}\right|_{t=0}\left\langle\mu, A d_{e^{t x}}(y)\right\rangle \\
& =\left\langle\mu,\left.\frac{d}{d t}\right|_{t=0} A d_{e^{t x}}(y)\right\rangle=\left\langle\mu, a d_{x}(y)\right\rangle=\langle\mu,[x, y]\rangle=\left\langle a d_{x}^{*} \mu, y\right\rangle .
\end{aligned}
$$

So, $\mu(0)=\mu$ and $\mu^{\prime}(0)=a d_{x}^{*}(\mu)$. Thus,

$$
T_{\mu} \mathscr{O}=\left\{a d_{x}^{*}(\mu) \mid x \in \mathfrak{g}\right\}
$$

\subsection{Isotropy Lie algebra}

It is possible to define, algebraically, the cotangent space [Warner 1983] in a point $x \in M$

$$
T_{x}^{*} M=\frac{m_{x}}{m_{x}^{2}}
$$

where $m_{x}$ is the ideal of germ of functions that vanish in $x$. If $M$ is a Poisson manifold in a way that the Rank of the Poisson structure is zero at $\mathrm{x}, m_{x}^{2}$ is an ideal of the Lie algebra $\left(C^{\infty}(M),\{\cdot, \cdot\}\right)$. Indeed, take $f g \in m_{x}^{2}$, by Leibniz rule, $\{h, f g\}=f\{h, g\}+\{h, f\} g$, since $\{h, g\}(x)=0$ for all $f \in C^{\infty}(M),\{h, f g\} \in m_{x}^{2}$. In this way, $T_{x}^{*} M$ has a Lie algebra structure, and $T_{x} M=\left(T_{x}^{*} M\right)^{*}$ has a linear Poisson structure. 
Explicitly, this structure is given by

$$
\left[d_{x} f, d_{x} g\right]:=d_{x}\{f, g\} .
$$

This Lie algebra is called the isotropy Lie algebra at $x$ and it is denoted by $\mathfrak{g}_{x}$. In a local coordinate $\left(y^{i}\right)$ we have the structure constants

$$
\left[d_{x} y^{i}, d_{x} y^{j}\right]=\sum_{k} \frac{\partial \Pi_{i j}}{\partial y^{k}}(x) d y^{k}=\sum_{k} c_{i, j}^{k} d y^{k}
$$

This definition will play an important role in the linearization problem presented in the chapter 3.

\subsection{Complete Poisson manifold}

Let us turn back to the example (36). Since $\mathbb{R}^{2 n}$ with that Poisson structure given by

$$
\Pi=\sum_{i=1}^{n} \frac{\partial}{\partial q^{i}} \wedge \frac{\partial}{\partial p^{i}}
$$

is a symplectic manifold, it is the only symplectic leaf itself. On the other hand, in $\mathbb{R}^{n}$ with the trivial Poisson structure, every vector field is null, so any point is a symplectic leaf. This simple example shows us that a Poison morphism does not necessarily map a symplectic leaf into a symplectic leaf. To avoid this kind of situation it is convenient to define the notion of a complete Poisson map.

Definition 55. Let $f$ be a function on a Poisson manifold $M$. We call $f$ complete if the Hamiltonian vector field $X_{f}$ is a complete vector field, i. e., its flow is globally defined.

If $f$ is a Casimir function of $(M, \Pi)$, then $X_{f}$ is a null vector field, so the flow of $X_{f}$ is a point and is defined for all times. Thus, $f$ is complete vector field.

Definition 56. A Poison manifold is called complete if every Hamiltonian vector field on $M$ is complete.

On [Marsden and Ratiu 2002] it is presented the following proposition - from the original reference, corollary 4.1.20:

Proposition 57. A $C^{r}$ vector field with compact support on a manifold $M$ is complete. In particular, a $C^{r}$ vector field defined on a compact manifold is complete.

In our case, we consider $r=\infty$. The above proposition ensures to us that every support compact function on an arbitrary Poisson manifold is complete. In particular, it ensures that every compact Poisson manifold is complete. Now we can define the notion of complete Poisson maps. 
Definition 58. A Poisson map $\phi: M \rightarrow N$ will be called complete if the pullback by $\phi$ of every complete function is complete.

The next proposition tells us that the definition of a complete Poisson map is sufficient to exclude the problem presented at the beginning of the section.

Proposition 59. The image of a complete Poisson map is a union of symplectic leaves.

The following proof is presented in [Silva and Weinstein 1999].

Proof. First, one can see that the condition of "the pullback by $\phi$ of every complete function is complete" could be replaced by "for each $f \in C^{\infty}(M)$, if $X_{f}$ is a complete vector field then $X_{\Phi * f}$ is also complete".

Now, let $\phi(x) \in N$, it is possible to find a piecewise Hamiltonian composed of complete vector fields $X_{h}$ 's. By definition, every pullback $X_{\phi * h}$ is a complete vector field. Hence, we can integrate them without restriction, and their flows provide a path on $M$. The image of this path is the original one. So, we conclude that any point of the symplectic leaf of $\phi(x)$ is contained in the image of $\phi$.

\subsection{Symplectic realization}

Definition 60. Let $(M, \Pi)$ be a Poisson manifold. Then, a full symplectic realization of $M$ is a symplectic manifold $(S, \omega)$ along with a surjective submersion $\mu: S \rightarrow M$ such that it is a Poisson map.

In the paper [Weinstein 1983], Weinstein makes a distinction between symplectic realizations that are surjective submersions and the ones which are not required to be a surjective submersion. He called the first ones by full symplectic realizations and the second ones just by symplectic realizations.

Although the second case is a more general definition, it includes a lot of trivial answers. For example, the inclusion of a symplectic leaf is a symplectic realization. For our purposes, from now on, we will consider just the case of full symplectic realization, and, as made by others, ignore the term "full" [Dufour and Zung 2006, Vaisman 2012].

Definition 61. A symplectic realization of a Poisson manifold $M$ is called complete if the surjective submersion $\mu: S \rightarrow M$ is complete.

The existence of complete symplectic realization is introduced in [Crainic and Fernandes 2004] as sufficient and necessary condition to the integrability of the $T^{*} M$ Lie algebroid. 
Example 62. Let $\mathbb{R}^{2}$ equipped with the bracket $\{x, y\}=0$ and let the $\mathbb{R}^{4}$ equipped with the Darboux coordinate $\left(q_{1}, q_{2}, p_{1}, p_{2}\right)$. The map $\mu: \mathbb{R}^{4} \rightarrow \mathbb{R}^{2}$ defined by $\mu\left(q_{1}, q_{2}, p_{1}, p_{2}\right)=$ $\left(q_{1}, p_{1} q_{1}+q_{2}\right)$ is a surjective submersion Poisson map, so it is a symplectic realization. 



\section{LIE ALGEBROID AND LIE GROUPOID}

The concept of a Lie algebroid can be seen as an infinitesimal part of a Lie groupoid, which will be discussed later. This concept unifies several structures, such as foliated manifolds, Poisson manifolds and infinitesimal actions of Lie algebras on a manifold. The aim of this chapter it to present several definitions and results of Lie algebroid and Lie groupoid theory.

First of all, we review the theory for an abstract Lie algebroid and we present some examples. Next, we present the relation between Poisson geometry and Lie algebroids. In section (3.2.2), we prove the splitting theorem for Lie algebroids. At last, we introduce some basic concepts of the Lie groupoid theory. In Section 3.2.1 we introduce the isotropy algebras and the foliation induced by the anchor map. After that we present the analogue of the Weinstein Splitting Theorem for Lie algebroids. We present the concept of Lie algebroid cohomology in the Section 3.2.3. In the subsequent sections we present the Lie groupoid theory and its relation with Lie algebroid theory.

\subsection{Lie algebroid}

In order to define a Lie algebroid, we start first introduce the concept of an anchored vector bundle.

Definition 63. An anchored vector bundle is a vector bundle $A \stackrel{\operatorname{proj}_{A}}{\longrightarrow} M$ together with a vector bundle map \# : $A \rightarrow T M$ called the anchor. The induced map $\Gamma(A) \rightarrow \mathfrak{X}(M)$ defined pointwise by $\#(X)\left(x_{0}\right)=\#\left(X\left(x_{0}\right)\right)$, will be denote simply by \#.

Roughly speaking, an anchored vector bundle generalizes the idea of the tangent bundle. Precisely, the anchor identify every $\gamma \in \Gamma(A)$ with the derivation $\#(\gamma)$ on $C^{\infty}(M)$.

Since a Lie algebroid can be thought as a generalization of Lie algebra and of tangent bundle to a given manifold, we need to give a Lie algebra structure on $\Gamma(A)$, the space of global 
sections of an anchored vector bundle. Moreover, one needs that the anchor map preserves the Lie structure on $\Gamma(A)$ and on $\Gamma(T M)$. More precisely:

Definition 64. A Lie algebroid $(A,[\cdot, \cdot], \#)$ over $M$ is an anchored vector bundle along with a Lie bracket $[\cdot, \cdot]$ in the space of smooth section $\Gamma(A)$. The Lie bracket satisfies the following Leibniz rule

$$
[\alpha, f \beta]=f[\alpha, \beta]+\mathscr{L}_{\#(\alpha)}(f) \beta, \forall \alpha, \beta \in \Gamma(A) \text { and } f \in C^{\infty}(M)
$$

Note that, by skew-symmetry and Leibniz rule, one has

$$
[f \alpha, \beta]=-[\beta, f \alpha]=f[\alpha, \beta]-\mathscr{L} \# \beta(f) \alpha
$$

Proposition 65. If $(A,[,] . \#)$ is a Lie algebroid, then the anchor $\#: \Gamma(A) \mapsto \mathfrak{X}(M)$ is a homeomorphism of Lie algebra, i.e.,

$$
\#[\alpha, \beta]=[\# \alpha, \# \beta] \forall \alpha, \beta \in \Gamma(A)
$$

Proof. Jacobi identity and the Leibniz rule imply:

$$
\begin{aligned}
0= & {[[\alpha, \beta], f \gamma]+[[\beta, f \gamma], \alpha]+[[f \gamma, \alpha], \beta] } \\
= & f[[\alpha, \beta], \gamma]+\#[\alpha, \beta](f) \gamma+[f[\beta, \gamma]+\# \beta(f) \gamma, \alpha]+[f[\gamma, \alpha]-\# \alpha(f)[\gamma, \beta] \\
= & f[[\alpha, \beta], \gamma]+\#[\alpha, \beta](f) \gamma \\
& +f[[\beta, \gamma], \alpha]-\# \alpha(f)[\beta, \gamma]+\# \beta(f)[\gamma, \alpha]-\# \alpha(\# \beta(f) \gamma \\
& +f[[\gamma, \alpha], \beta]-\# \beta(f)[\gamma, \alpha]+\# \alpha(f)[\gamma, \beta]-\# \beta(\# \alpha(f)) \gamma \\
= & ((\#[\alpha, \beta]-[\# \alpha, \# \beta])(f)) \gamma
\end{aligned}
$$

Since $\alpha, \beta, \gamma$ and $f$ are arbitrary, we have $\#[\alpha, \beta]=[\# \alpha, \# \beta]$.

Example 66. I. Any Lie algebra is a Lie algebroid over a point, where the anchor is the null function.

II. Any vector bundle with the null anchor and trivial bracket is a Lie algebroid.

III. The tangent bundle of any smooth manifold with the standard Lie bracket of vector fields and the identity map as anchor is a Lie algebroid.

A less trivial example is

Example 67. Let $\omega$ be a closed 2-form on $M$. Thus, we give a Lie algebroid structure on $M$, called $A_{\omega}$, defined by 
- The vector bundle structure

$$
A_{\omega}=T M \otimes(M \times \mathbb{R}) .
$$

- The anchor map, which is the projection on the first component.

- The Lie bracket $\Gamma\left(A_{\omega}\right) \simeq \mathfrak{X}(M) \times C^{\infty}(M)$, which is defined by

$$
[(X, f),(Y, g)]=\left([X, Y], \mathscr{L}_{X}(g)-\mathscr{L}_{Y}(f)+\omega(X, Y)\right) .
$$

Let $X, Y, Z \in \mathfrak{X}(M)$ and $f, g, h \in C^{\infty}(M)$, so we can prove

- The Jacobi Identity:

$$
\begin{aligned}
{[X+f,[Y+g, Z+h]]=} & {\left[X+f,[Y, Z]+\mathscr{L}_{Y}(h)-\mathscr{L}_{Z}(g)+w(Y, Z)\right.} \\
= & {[X,[Y, Z]]+\mathscr{L}_{X}\left(\mathscr{L}_{Y}(h)\right)-\mathscr{L}_{X}\left(\mathscr{L}_{Z}(g)\right) } \\
& +\mathscr{L}_{X}(\omega(Y, Z))-\mathscr{L}_{[Y, Z]}(f)+\omega(X,[Y, Z]) \\
{[Y+g,[Z+h, X+f]]=} & {\left[Y+g,[Z, X]+\mathscr{L}_{Z}(f)-\mathscr{L}_{X}(h)+w(Z, X)\right.} \\
= & {[Y,[Z, X]]+\mathscr{L}_{Y}\left(\mathscr{L}_{Z}(f)\right)-\mathscr{L}_{Y}\left(\mathscr{L}_{X}(h)\right) } \\
& +\mathscr{L}_{Y}(\omega(Z, X))-\mathscr{L}_{[Z, X]}(g)+\omega(Y,[Z, X]) \\
& \\
{[Z+h,[X+f, Y+g]]=} & {\left[Z+h,[X, Y]+\mathscr{L}_{X}(g)-\mathscr{L}_{Y}(f)+w(X, Y)\right.} \\
= & {[Z,[X, Y]]+\mathscr{L}_{Z}\left(\mathscr{L}_{X}(g)\right)-\mathscr{L}_{Z}\left(\mathscr{L}_{Y}(f)\right) } \\
& +\mathscr{L}_{Z}(\omega(X, Y))-\mathscr{L}_{[X, Y]}(h)+\omega(Z,[X, Y])
\end{aligned}
$$

thus, we have

$$
\begin{aligned}
{[X} & +f,[Y+g, Z+h]]+[Y+g,[Z+h, X+f]]+[Z+h,[X+f, Y+g]]= \\
= & {[X,[Y, Z]]+[Y,[Z, X]]+[Z,[X, Y]] } \\
& +\mathscr{L}_{[X, Y]}(h)+\mathscr{L}_{[Z, X]}(g)+\mathscr{L}_{[Y, Z]}(f)-\mathscr{L}_{[X, Y]}(h)-\mathscr{L}_{[Z, X]}(g)-\mathscr{L}_{[Y, Z]}(f) \\
& +\mathscr{L}_{X}(\omega(Y, Z))-\mathscr{L}_{Y}(\omega(X, Z))+\mathscr{L}_{Z}(\omega(X, Y)) \\
& -\omega([X, Y], Z)-\omega([Y, Z], X)+\omega([X, Z], Y) \\
= & d \omega(x, y, z)
\end{aligned}
$$

Since $\omega$ is closed, $d \omega=0$ and this bracket satisfies Jacobi.

- Leibniz Rule

$$
\begin{aligned}
{[X+f, h Y+h g] } & =[X, h Y]+\mathscr{L}_{X}(h g)-\mathscr{L}_{h Y}(f)+\omega(X, h Y) \\
& =\mathscr{L}_{X}(g) Y+g \mathscr{L}_{X}(Y)+\mathscr{L}_{X}(g) h+g \mathscr{L}_{X}(h)-g \mathscr{L}_{Y}(f)+f \omega(X, Y) \\
& =g\left([X, Y]+\mathscr{L}_{X}(h)-\mathscr{L}_{Y}(f)+w(X, Y)\right)+\mathscr{L}_{X}(g)(Y+h)
\end{aligned}
$$


Example 68 (Transformation Algebroid Associated to the Infinitesimal Action). Let $M$ be a manifold equipped with an infinitesimal action of a Lie algebra $\mathfrak{g}$, i.e, a Lie algebra homomorphism $\phi: \mathfrak{g} \rightarrow \mathfrak{X}(M)$. The vector bundle $M \times \mathfrak{g}$ over $M$ has the following Lie algebroid structure, with anchor and the Lie bracket given by

$$
\begin{aligned}
& \#(x, \xi)=\phi(\xi)_{x} \\
& {[u, v](x)=[u(x), v(x)]+(\phi(u(x))(v))(x)-(\phi(v(x))(x))(x),}
\end{aligned}
$$

where $u, v \in C^{\infty}(M, \mathfrak{g}) \cong \Gamma(M, \mathfrak{g} \times M)$ and $x \in M$.

Definition 69. Let $\left(A_{1}, \#_{1},[\cdot, \cdot]_{1}\right)$ and $\left(A_{2}, \#_{2},[\cdot, \cdot]_{2}\right)$ be Lie algebroids over the same manifold $M$. A Lie algebroid morphism over the same manifold is a vector bundle morphism $\Phi: A_{1} \rightarrow A_{2}$, e. g., a functions $\Phi: A_{1} \rightarrow A_{2}$ such that, for every $x \in M$, the map $\operatorname{proj}_{1}^{-1}(x) \rightarrow \operatorname{proj}_{2}^{-1}(x)$ induced by $F$ is a linear map satisfying

$$
\begin{aligned}
& \text { I } \#_{2} \circ \Phi=\#_{1} \\
& \text { II } \Phi\left([\alpha, \beta]_{1}\right)=[\Phi(\alpha), \Phi(\beta)]_{2} \text {, for all } \alpha, \beta \in A_{1} .
\end{aligned}
$$

A Lie algebroid isomorphism is a morphism that is invertible.

Remark 70. A general definition of Lie algebroid morphism is given in [Crainic and Fernandes 2011], but in this monograph we are just interested in morphism of Lie algebroids over the same manifold.

\subsection{Relation between Lie algebroid theory and Poisson geometry}

The first way to relate the Poisson geometry with Lie algebroid theory is given by the following important example.

Proposition 71. Let $(M,\{\cdot, \cdot\})$ be a Poisson manifold. Then, there exists a $\mathbb{R}$-bilinear, skewsymmetric operation $[\cdot, \cdot]: \Gamma\left(T^{*} M\right) \times \Gamma\left(T^{*} M\right) \rightarrow \Gamma\left(T^{*} M\right)$ defined by

$$
[\alpha, \beta]=\mathscr{L}_{\# \alpha}(\beta)-\mathscr{L}_{\# \beta}(\alpha)-d(\Pi(\alpha, \beta))
$$

So we are ready to give a Lie algebroid structure to every cotangent bundle of a Poisson manifold.

Proposition 72. The cotangent bundle of a Poisson manifold $M$, with the bracket above and the Poisson bundle morphism \#, given by the contraction with $\Pi$, is a Lie algebroid over $M$. It is call this the cotangent Lie algebroid of the Poisson manifold $(M, \Pi)$. 
Proof. To check the Jacobi identity:

$$
\begin{aligned}
& {[\alpha,[\beta, \gamma]]=\left[\alpha, \mathscr{L}_{\# \beta}(\gamma)-\mathscr{L}_{\# \gamma}(\beta)-d \Pi(\beta, \gamma)\right]} \\
& =\mathscr{L}_{\# \alpha}\left(\mathscr{L}_{\# \beta}(\gamma)\right)-\mathscr{L}_{\# \alpha}\left(\mathscr{L}_{\# \gamma}(\beta)\right)-\mathscr{L}_{\# \alpha}(d \Pi(\beta, \gamma)) \\
& -\mathscr{L}_{\# \mathscr{L}_{\# \beta}(\gamma)}(\alpha)+\mathscr{L}_{\# \mathscr{L}_{\# \gamma}(\beta)}(\alpha)+\mathscr{L}_{\# d \Pi(\beta, \gamma)}(\alpha) \\
& d\left(\Pi\left(\alpha, \mathscr{L}_{\# \beta}(\gamma)\right)+d\left(\Pi\left(\alpha, \mathscr{L}_{\# \gamma}(\beta)\right)+d(\Pi(\alpha, d \Pi(\beta, \gamma))\right.\right. \\
& {[\beta,[\gamma, \alpha]]=\left[\beta, \mathscr{L}_{\# \gamma}(\alpha)-\mathscr{L}_{\# \alpha}(\gamma)-d \Pi(\gamma, \alpha)\right]} \\
& =\mathscr{L}_{\# \beta}\left(\mathscr{L}_{\# \gamma}(\alpha)\right)-\mathscr{L}_{\# \beta}\left(\mathscr{L}_{\# \alpha}(\gamma)\right)-\mathscr{L}_{\# \beta}(-d \Pi(\gamma, \alpha)) \\
& -\mathscr{L}_{\# \mathscr{L}_{\# \gamma}(\alpha)}(\beta)+\mathscr{L}_{\# \mathscr{L}_{\# \alpha}(\gamma)}(\beta)+\mathscr{L}_{\# d \Pi(\gamma, \alpha)}(\beta) \\
& -d\left(\Pi\left(\beta, \mathscr{L}_{\# \gamma}(\alpha)\right)+d\left(\Pi\left(\beta, \mathscr{L}_{\# \alpha}(\gamma)\right)+d(\Pi(\beta, d \Pi(\gamma, \alpha))\right.\right. \\
& {[\gamma,[\alpha, \beta]]=\left[\gamma, \mathscr{L}_{\# \alpha}(\beta)-\mathscr{L}_{\# \beta}(\alpha)-d \Pi(\alpha, \beta)\right]} \\
& =\mathscr{L}_{\# \gamma}\left(\mathscr{L}_{\# \alpha}(\beta)\right)-\mathscr{L}_{\# \gamma}\left(\mathscr{L}_{\# \beta}(\alpha)\right)-\mathscr{L}_{\# \gamma}(d \Pi(\alpha, \beta)) \\
& -\mathscr{L}_{\# \mathscr{L}_{\# \alpha}(\beta)}(\gamma)+\mathscr{L}_{\# \mathscr{L}_{\# \beta}(\alpha)}(\gamma)+\mathscr{L}_{\# d \Pi(\alpha, \beta)}(\gamma) \\
& d\left(\Pi\left(\gamma, \mathscr{L}_{\# \alpha}(\beta)\right)+d\left(\Pi\left(\gamma, \mathscr{L}_{\# \beta}(\alpha)\right)+d(\Pi(\gamma, d \Pi(\alpha, \beta))\right.\right.
\end{aligned}
$$

So, we have that

$$
\begin{aligned}
{[\alpha,[\beta, \gamma]]+[\beta,[\gamma, \alpha]]+[\gamma,[\beta, \alpha]]=} & \mathscr{L}_{[\# \alpha, \# \beta]}(\gamma)+\mathscr{L}_{[\# \beta, \# \gamma]}(\alpha)+\mathscr{L}_{[\# \gamma, \# \alpha]}(\beta) \\
& -\mathscr{L}_{\#[\beta, \gamma]}(\alpha)-\mathscr{L}_{\#[\alpha, \gamma]}(\beta)-\mathscr{L}_{\#[\alpha, \beta]}(\gamma) \\
& +d(\Pi(\alpha, d \Pi(\beta, \gamma)+\Pi(\beta, d \Pi(\gamma, \alpha))+\Pi(\gamma, d \Pi(\alpha, \beta)))
\end{aligned}
$$

since, $\#[\alpha, \beta]=[\# \alpha, \# \beta]$ for all $\alpha, \beta \in \Gamma(A)$ and

$$
d(\Pi(\alpha, d \Pi(\beta, \gamma)+\Pi(\beta, d \Pi(\gamma, \alpha))+\Pi(\gamma, d \Pi(\alpha, \beta)))=0
$$

we get

$$
[\alpha,[\beta, \gamma]]+[\beta,[\gamma, \alpha]]+[\gamma,[\beta, \alpha]]=0
$$

To check Leibniz's rule:

$$
\begin{aligned}
{[\alpha, f \beta] } & =\mathscr{L}_{\# \alpha}(f \beta)-\mathscr{L}_{\# f \beta}(\alpha)-d(\Pi(\alpha, f \beta)) \\
& =f i_{\# \alpha} \beta+d f i_{\# \alpha} \beta+f d\left(i_{\# \alpha} \beta\right)-f \mathscr{L}_{\# \beta}(\alpha)-d f \wedge i_{\# \beta} \alpha-d f \Pi(\alpha, \beta)-f \Pi(\alpha, \beta) \\
& =f\left(i_{\# \alpha} \beta+d\left(i_{\# \alpha} \beta\right)-\mathscr{L}_{\# \beta}(\alpha)-\Pi(\alpha, \beta)\right)+d f i_{\# \alpha} \beta-d f i_{\# \beta} \alpha-d f \Pi(\alpha, \beta) \\
& =f\left(\mathscr{L}_{\# \alpha}(\beta)-\mathscr{L}_{\# \beta}(\alpha)-\Pi(\alpha, \beta)\right)+d f\left(i_{\# \alpha} \beta-i_{\# \beta} \alpha-\Pi(\alpha, \beta)\right)
\end{aligned}
$$


We saw in Section 2.3.2 that the dual space of any Lie algebra has a linear Poisson structure. We have an analogous result for Lie Algebroid, but in this case, the Poisson structure is fiber-wise linear. In this section we follow mostly [MEINRENKEN 2017, Courant 1990, Dufour and Zung 2006].

Let $E \stackrel{\pi}{\rightarrow} M$ be a vector bundle. Let $\mu_{t}: E \rightarrow E,(x, v) \mapsto(x, t v)$ be the fiber-wise scalar multiplication by $t \in \mathbb{R}$. For every $t \neq 0$ this application is a diffeomorphism.

Definition 73. A multivector field $v \in \mathfrak{X}^{k}(E)$ is called fiber-wise linear if it is homogeneous of degree $1-k$, that is,

$$
\mu_{t}^{*}(v)=t^{(1-k)} v, t \neq 0
$$

Let $\left(U, x^{1}, \ldots, x^{n}\right)$ be a chart on $M$ and let $\left(y^{1}, \ldots, y^{m}\right)$ be the fiber coordinates, hence, we have a local coordinate $\left(\pi^{-1} U, x^{1}, \ldots, x^{n}, y^{1}, \ldots, y^{m}\right)$ such that, a fiber-wise linear multivector field has the form

$$
v=\sum_{\left(i_{1}, \ldots, i_{k}\right)} \sum_{j} c_{j}^{\left(i_{1}, \ldots, i_{k}\right)} y^{j} \frac{\partial}{\partial y^{i_{1}}} \wedge \ldots \wedge \frac{\partial}{\partial y^{i_{k}}}+\sum_{\left(i_{1}, \ldots, i_{k-1}\right)} \sum_{j} a^{\left(i_{1}, \ldots, i_{k-1}, r\right)} \frac{\partial}{\partial x^{r}} \wedge \frac{\partial}{\partial y^{i_{1}}} \wedge \ldots \wedge \frac{\partial}{\partial y^{i_{k-1}}},
$$

where $c_{j}^{\left(i_{1}, \ldots, i_{k}\right)}$ and $a^{\left(i_{1}, \ldots, i_{k-1}, r\right)}$ are smooth functions on $U$. We are interested in fiber-wise linear bi-vector fields.

Let $p: E^{*} \rightarrow M$ the dual vector bundle of $E$. Every $\alpha \in \Gamma(E)$ can be seen as a smooth function, $\phi_{\alpha}$ on $E^{*}, \phi_{\alpha}(x, v) \mapsto \alpha(v)(x)$. Of course, this function is linear on each fiber of $E$. We have another subset of smooth function on $E^{*}$, the functions which are constant on the fibers. It is clear that this functions are in one-to-one correspondence with the smooth function on the base $M$. In fact, if we have a function $f \in C^{\infty}\left(E^{*}\right)$ that is constant on the fibers, $f(x)=f\left(v_{x}\right) \in C^{\infty}(M)$, where $v_{x} \in E_{x}^{*}$. On the other hand, let $f \in C^{\infty}(M)$, so $f \circ p \in C^{\infty}\left(E^{*}\right)$ is constant on the fibers.

Now we are ready to prove the following theorem.

Theorem 74. For any Lie algebroid $A \rightarrow M$, the total space of dual bundle $p: A^{*} \rightarrow M$ has a unique Poisson bracket such that for all $\alpha, \beta \in \Gamma(A)$ and $f, g \in C^{\infty}(M)$,

$$
\begin{aligned}
\left\{\phi_{\alpha}, \phi_{\beta}\right\} & =\phi_{[\alpha, \beta]}, \\
\left\{\phi_{\alpha}, f \circ p\right\} & =\#(\alpha)(f) \circ p, \\
\{f \circ p, g \circ p\} & =0 .
\end{aligned}
$$

Proof. Let $\left.A\right|_{U}=U \times \mathbb{R}^{m}$ be a local bundle trivialization over open subset $U \subset M$, let $\left(e_{1}, \ldots, e_{m}\right)$ be the corresponding basis of sections of $A$, and let $\left(x^{1}, \ldots, x^{n}\right)$ be the local coordinates on $U$. First of all, note that the differentials of the functions $y_{i}=\phi_{e_{i}}$ and $x^{j} y_{i}=\phi_{x^{j} y_{i}}$ span $T^{*}\left(A^{*}\right)$ everywhere, except where all $y^{j}$ vanish. Thus,

$$
\Pi\left(d \phi_{\alpha}, d \phi_{\beta}\right)=\phi_{\alpha, \beta}
$$


define a bi-vector field on $A^{*}$. In local coordinate, it look like

$$
\Pi=\frac{1}{2} \sum_{i, j}\left[e_{i}, e_{j}\right] \frac{\partial}{\partial y_{i}} \wedge \frac{\partial}{\partial y_{j}}+\sum_{i, j}\left(\#\left(e_{i}\right)\left(x^{i}\right)\right) \frac{\partial}{\partial y_{i}} \wedge \frac{\partial}{\partial x^{j}} .
$$

The Jacobi identity is trivially verified for all functions except for the case when two functions are fiber-wise linear, $\phi_{\alpha}, \phi_{\beta}$, and one is constant on the fibers, $f \circ p$.

$$
\left\{\left\{\phi_{\alpha}, \phi_{\beta}\right\}, f \circ p\right\}=\left\{\phi_{[\alpha, \beta]}, f \circ p\right\}=\#([\alpha, \beta])(f)=\{\alpha,\{\beta, f\}\}-\{\beta,\{\alpha, f \circ p\}\} .
$$

Example 75. In Section (2.3.3) we saw the fiber-wise linear Poisson structure defined on the dual of the cotangent bundle with the Lie algebroid structure given in the Section (3.2).

Example 76. In this example we describe the fiber-wise linear Poisson structure on $\mathbb{L}_{\omega}$. Let $x_{1}, \ldots, x_{m}$ be local coordinates on $M$. We have the local frame $\left(p_{1}, \ldots, p_{m}, s, x_{1}, \ldots, x_{m}\right)$, for $T M \oplus(M \times \mathbb{R})$, where $p_{i}=\frac{\partial}{\partial x_{i}}$ and $s=1$ is the constant function. Thus, we have

$$
\begin{aligned}
& {\left[p_{i}, p_{j}\right]=\omega\left(p_{i}, p_{j}\right)} \\
& {\left[p_{i}, s\right]=\mathscr{L}_{p_{i}}(s)} \\
& \#\left(p_{i}\right)\left(x_{j}\right)=\frac{\partial x_{i}}{\partial x_{j}}=\delta_{i}^{j} \\
& \#(s)\left(x_{i}\right)=\#(s)\left(x_{i}\right)=0
\end{aligned}
$$

where $\delta_{i}^{j}=1$, if $i=j$, and zero otherwise. Then, in these local coordinates the bivector is given by

$$
\Pi=\sum_{i, j} \omega\left(p_{i}, p_{j}\right) \frac{\partial}{\partial p_{i}} \wedge \frac{\partial}{\partial p_{j}}+\sum_{i}\left(\mathscr{L}_{p_{i}}\left(s_{x}\right) \frac{\partial}{\partial p_{i}} \wedge \frac{\partial}{\partial s_{x}}\right)+\sum_{i} \frac{\partial}{\partial p_{i}} \wedge \frac{\partial}{\partial x_{i}}
$$

\subsubsection{Isotropy algebras and characteristic foliation of a Lie algebroid}

Let $(A,[\cdot, \cdot], \#)$ be a Lie algebroid over a manifold $M$. Given $z \in M$, we will denote by $A_{z}$ the fiber of $A$ over $z$, and by $\mathrm{Ker}_{z}$ the kernel of the anchor map

$$
\#_{z}: A_{z} \rightarrow T_{z} M
$$

The kernel $\mathrm{Ker}_{z}$ has a natural Lie algebra structure, defined as the following. For any $\alpha_{z}, \beta_{z} \in$ $\operatorname{Ker}_{z}$, let $\alpha, \beta$ arbitrary sections of $A$ such that $\alpha(z)=\alpha_{z}$ and $\beta(z)=\beta_{z}$, so we set

$$
\left[\alpha_{z}, \beta_{z}\right]=[\alpha, \beta](z)
$$

The above bracket is well defined. Indeed, take another section of $A, \tilde{\alpha}$, with $\tilde{\alpha}(z)=\alpha_{z}$, then, locally, there are functions $f_{1}, \ldots, f_{n}$ on $M$ which vanish at $z$ and a basis $\left(\alpha_{1}, \ldots, \alpha_{n}\right)$ of $A$ such that $\tilde{\alpha}-\alpha=\sum_{i=1}^{n} f_{i} \alpha_{i}$. By the Leibniz rule, we have $[\tilde{\alpha}, \beta](z)-[\alpha, \beta](z)=\sum_{i=1}^{n} f_{i}(z)\left[\alpha_{i}, \beta\right](z)-$ $\sum_{i=1}^{n}(\# \beta)\left(f_{i}\right)(z) \alpha_{i}(z)=0$, because $f_{i}(z)=0$ and $\# \beta(z)=0$. 
Definition 77. For any $z \in M$ we call the Lie algebra

$$
\mathfrak{g}_{z}(A):=\operatorname{Ker\# }_{z}
$$

the isotropy Lie algebra at $z$

The distribution $\mathscr{C}: x \in M \mapsto \mathscr{C}_{x}=\operatorname{Im}_{x}$ is called the characteristic distribution of $A$, and dimension of $\operatorname{Im}_{x}$ is called the Rank of \# at $x$.

Let $\left(\alpha_{1}, \ldots, \alpha_{m}\right)$ be a local basis of sections of a Lie algebroid $A$, then we have that $\left[\alpha_{i}, \alpha_{j}\right]=\sum_{k} c_{i j}^{k} \alpha_{k}$. Since the anchor is a morphism of Lie algebras we get

$$
\left[\#\left(\alpha_{i}\right), \#\left(\alpha_{j}\right)\right]=\#\left(\left[\alpha_{i}, \alpha_{j}\right]\right)=\sum_{k} c_{i j}^{k} \#\left(\alpha_{k}\right) .
$$

Thus, the characteristic distribution is integrable.

\subsubsection{Splitting theorem}

Analogue of the Weinstein Splitting Theorem for Poisson manifolds, for all Lie algebroid there are some local coordinates and sections that simplify the Lie algebroid structure.

Theorem 78. Let $(A,[\cdot, \cdot], \#)$ be a Lie algebroid over $M$. Then $A$ can be decomposed locally into the direct product of a tangent algebroid and a Lie algebroid whose Rank at origin is 0 . More precisely, if the Rank of \# at a point $z$ of $M$ is q, then there is a system of local coordinates $\left(x_{1}, \ldots, x_{q}, y_{1}, \ldots y_{s}\right)$ on $M$ centered at $z$, and a basis of local sections $\left(\alpha_{1}, \ldots, \alpha_{q}, \beta_{1}, . ., \beta_{r}\right)$ of $A$ over a neighborhood of $z$, such that the following conditions are satisfied for all possibles indices:

$$
\begin{gathered}
{\left[\alpha_{i}, \alpha_{j}\right]=0, \# \alpha_{i}=\frac{\partial}{\partial x_{i}},} \\
{\left[\beta_{i}, \alpha_{j}\right]=0, \# \beta_{i}\left(x_{j}\right)=0, \mathscr{L}_{\frac{\partial}{\partial x_{i}}} \# \beta_{i}=0,} \\
{\left[\beta_{i}, \beta_{j}\right]=\sum_{k} f_{i j}^{k}(y) \beta_{k},}
\end{gathered}
$$

where $f_{i j}^{k}(y)$ are functions which depend only on the variables $y=\left(y_{1}, \ldots, y_{s}\right)$.

Proof. We will prove by induction on the Rank\# $\#_{x}=q$. When $q=0$, we are done, thus, we will assume that $q>0$. Suppose that, for any integer $d$ such that $0 \leq d<q$ we have found a local coordinate system $\left(x_{1}, . ., x_{d}, y_{1}, \ldots, y_{s+q-d}\right)$ of $M$, and a basis of local sections $\left(\alpha_{1}, \ldots, \alpha_{d}, \beta_{1}, \ldots, \beta_{r+q-d}\right)$ satisfying (3.2.3), (3.2.4) and (3.2.5).

Since $d<q$, there must be some $\beta_{i}$ such that $\#_{z}(\beta) \neq 0$. Without loss of generality, we can assume that $i=1$. By the straightening theorem, it is possible to assume that $\# \beta_{1}=\frac{\partial}{\partial y_{1}}$. 
We want to find a coordinate system with the property that

$$
\left[\beta_{1}, \beta_{i}\right]=0, \forall i=2, \ldots,(r+q-d),
$$

so we replace $\beta_{i}$ by $\sum_{j=1}^{r+q-d} g_{i}^{j} \beta_{j}$, where the functions $g_{i}^{j}$ depend only on $\left(y_{1}, \ldots, y_{s+q-d}\right)$. By the Leibniz rule, the condition (3.2.6) is equivalent to

$$
\begin{aligned}
{\left[\beta_{1}, \quad \sum_{j=1}^{(r-q-d)} g_{1}^{j} \beta_{j}\right] } & =\sum_{j=1}^{(r-q-d)}\left[\beta_{1}, g_{1}^{j} \beta_{j}\right]=\sum_{j=1}^{(r-q-d)} g_{1}^{j}\left[\beta_{1}, \beta_{j}\right]+\frac{\partial g_{1}^{j}}{\partial y_{1}} \beta_{j} \\
& =\sum_{j=1}^{(r-q-d)}\left(\sum_{k=1}^{(r-q-d)} g_{i}^{j} f_{i, j}^{K} \beta_{k}+\frac{\partial g_{1}^{j}}{\partial y_{1}} \beta_{j}\right) .
\end{aligned}
$$

Since the equation (3.2.8) is an ordinary differential equation, we can find a coordinate system satisfying (3.2.6). The equation (3.2.6) implies that, for every $i=2, \ldots,(r+q-d)$

$$
\mathscr{L}_{\frac{\partial}{\partial y_{1}}}\left(\# \beta_{i}\right)=\left[\# \beta_{1}, \# \beta_{i}\right]=\#\left[\beta_{1}, \beta_{i}\right]=0
$$

Note that,

$$
\left[\beta_{1},\left(\# \beta_{i}\left(y_{1}\right)\right) \beta_{1}\right]=\# \beta_{i}\left(y_{1}\right)\left[\beta_{1}, \beta_{1}\right]+\mathscr{L}_{\# \beta_{1}}\left(\# \beta_{i}\left(y_{1}\right)\right) \beta_{1}=0 .
$$

Replacing $\beta_{i}$ by $\beta_{i}-\left(\# \beta_{i}\left(y_{1}\right)\right) \beta_{1}$ for each $i \geq 2$ we have that,

$$
\#\left(\beta_{i}-\left(\# \beta_{i}\left(y_{1}\right)\right) \beta_{1}\right)\left(y_{1}\right)=\left(\# \beta_{i}-\left(\# \beta_{i}\left(y_{1}\right)\right) \# \beta_{1}\right)\left(y_{1}\right)=\# \beta_{i}\left(y_{1}\right)-\# \beta_{i}\left(y_{1}\right) \frac{\partial y_{1}}{\partial y_{1}}=0
$$

At last,

$$
\left[\beta_{i}, \beta_{j}\right]=f_{i, j}^{1} \beta_{1}+\sum_{k=2}^{r+q-d} f_{i, j}^{k} \beta_{k}, i, j \geq 2 .
$$

Applying the anchor map to the (3.2.10), and valuing it on $y_{1}$, we get

$$
0=\left[\# \beta_{i}, \# \beta_{j}\right]\left(y_{1}\right)=\#\left(f_{i, j}^{1} \beta_{1}+\sum_{k=2}^{r+q-d} f_{i, j}^{k} \beta_{k}\right)=f_{i, j}^{1} .
$$

Thus, we have

$$
\left[\beta_{i}, \beta_{J}\right]=\sum_{k=2}^{r+q-d} f_{i, j}^{k} \beta_{k} .
$$

Now, reindexing the variables, we have the induction procedure.

Example 79. When we consider the tangent bundle of a manifold $M$ as a Lie algebroid, the anchor is the identity morphism, so the set of $\beta_{i}$ is empty. Let $\left(x_{i}, \ldots, x_{n}\right)$ be local coordinates to $M$. A clever guess is to set $\alpha_{i}=\frac{\partial}{\partial x_{i}}$ and, so, the theorem 78 holds.

Example 80. Let $A_{\omega}$ be the Lie algebroid associated to a closed 2-form as in the Example 67. We can set $\alpha_{i}=\left(\frac{\partial}{\partial x_{i}}, 0\right)$ and $\beta=(0,1)$. Let $(M, \Pi)$ be a Poisson manifold with split coordinates $\left(p_{1}, \ldots, p_{n}, q_{1}, \ldots, q_{n}, z_{1}, \ldots, z_{m-2 n}\right)$, so, setting $\alpha_{i}=d p_{i}, \alpha_{i+n}=d q_{i}$ and $\beta_{i}=z_{i}$, the theorem holds, again. 


\subsubsection{Lie algebroid cohomology}

This section is based on [Crainic 2003]. Aiming to define the Lie algebroid cohomology, we will introduce the concept of $A$-connection on a vector bundle. Let $E \rightarrow M$ be a $C^{\infty}$ vector bundle over $\mathrm{M}$ and let $(A,[\cdot, \cdot], \#)$ be a Lie algebroid over $M$. An $A$-connection $\nabla$ on $E$ is a bilinear map

$$
\begin{aligned}
\nabla: \Gamma(A) \times \Gamma(E) & \rightarrow \Gamma(E) \\
(\alpha, s) & \mapsto \nabla_{\alpha} s,
\end{aligned}
$$

such that, for all $f \in C^{\infty}(M), \alpha \in \Gamma(A)$ and $s \in \Gamma(E)$,

(i) $\nabla_{f \alpha} s=f \nabla_{\alpha} s$;

(ii) $\nabla_{\alpha}(f s)=f \nabla_{\alpha} s+\# \alpha(f) s$.

The curvature of the connection is the map

$$
\begin{aligned}
R_{\nabla}: & \Gamma(A) \times \Gamma(A) \rightarrow \operatorname{End}(\Gamma(E)) \\
R_{\nabla}(\alpha, \beta)(X) & =\nabla_{\alpha} \nabla_{\beta} X-\nabla_{\beta} \nabla_{\alpha} X-\nabla_{[\alpha, \beta]} X
\end{aligned}
$$

We say that an $A$-connection is flat if $R_{\nabla}=0$.

Remark 81. When the $A=T M$ we recover the usual notion of connection on vector bundles.

Definition 82. A represetation of a Lie algebroid $A$ over $M$ consists of a pair $(E, \nabla)$ of a vector bundle $E$ over $M$ endowed with a flat connection $\nabla$.

Given a representation $(E, \nabla)$ of a Lie algebroid $A$ we define a p-cochain on $A$ with values on $(E, \nabla)$ as a multilinear skew-symmetric map

$$
\begin{aligned}
c: \Gamma(A) \times \ldots \times \Gamma(A) & \rightarrow \Gamma(E) \\
\left(\alpha_{1}, \ldots, \alpha_{p}\right) & \mapsto c\left(\alpha_{1}, \ldots, \alpha_{p}\right)
\end{aligned}
$$

The space $C^{p}(A, E)$ of p-cochains coincides with the space of sections $\Gamma\left(\wedge^{p} A^{*} \otimes E\right)$. The family $C^{\bullet}(A, E)$ turns into a differential complex with the following differential operator $d: C^{p}(A, E) \rightarrow$ $C^{p+1}(A, E)$

$$
\begin{aligned}
d c\left(\alpha_{1}, \ldots, \alpha_{p+1}\right)= & \sum_{k=1}^{p+1}(-1)^{k+1} \nabla_{\alpha_{k}}\left(\omega\left(\alpha_{1}, \ldots, \hat{\alpha}_{k}, \ldots, \alpha_{p+1}\right)\right) \\
& +\sum_{k<l}(-1)^{k+l} \omega\left(\left[\alpha_{k}, \alpha_{l}\right], \ldots, \hat{\alpha}_{k} \ldots, \hat{\alpha}_{l} \ldots, \alpha_{p+1}\right) .
\end{aligned}
$$


We call cohomology of $A$ with coefficients in $E$, denoted $H^{\bullet}(A, E)$, the cohomology of the complex $\left(C^{\bullet}(A, E), d\right)$. When $E$ is the trivial line bundle with $\nabla_{\alpha} f=\mathscr{L}_{\# \alpha}(f)$, we use the notation $C^{\bullet}(A)$ and $H^{\bullet}(A)$. The Lie algebroid cohomology generalizes many cohomology theories [Crainic and Fernandes 2011].

Example 83 (Lie algebra cohomology). When $A=\mathfrak{g}$ is a Lie algebra, the vector bundle $E \rightarrow p$ is nothing but a vector space. A representation is merely a homomorphism $\rho: \mathfrak{g} \rightarrow \mathfrak{g l}(V)$, where $\mathfrak{g l}(V)$ is the Lie algebra of endomorphisms of $V$ with the usual commutator. Precisely, $\rho$ is linear and, for all $X, Y \in \mathfrak{g}$

$$
\rho([X, Y])=\rho(X) \rho(Y)-\rho(Y) \rho(X) .
$$

An p-chain will be a p-linear alternating mapping $c \in \operatorname{Hom}\left(\wedge^{q} \mathfrak{g}, V\right)$ and the differential operator will be defined by the formula

$$
\begin{aligned}
d(x)\left(\alpha_{1}, \ldots, \alpha_{p+1}\right)= & \sum_{k=1}^{p+1}(-1)^{k+1} \rho\left(\alpha_{k}\right)\left(c\left(\alpha_{1}, \ldots, \hat{\alpha}_{i}, \ldots, \alpha_{p+1}\right)\right) \\
& +\sum_{k<l}(-1)^{k+l} c\left(\left[\alpha_{k}, \alpha_{l}\right], \alpha_{1}, \ldots, \hat{\alpha}_{k}, \ldots, \hat{\alpha}_{l}, \ldots, \alpha_{p+1}\right) .
\end{aligned}
$$

This cohomology coincides with the Chevalley-Eilenberg complex [Chevalley and Eilenberg 1948].

Here we are interested in the next two Lie algebra cohomologies.

1. Let $V=\mathbb{R}$ and $\rho(X)=0$ for all $X \in \mathfrak{g}$. We denote the cohomology simply by $H^{\bullet}(\mathfrak{g})$ and the differential operator is reduced to

$$
d(\omega)\left(X_{1}, \ldots, X_{q+1}\right)=\sum_{i<j}(-1)^{i+j} \omega\left(\left[X_{i}, X_{j}\right], X_{1}, \ldots, \hat{X}_{i}, \ldots, \hat{X}_{j}, \ldots X_{q+1}\right) .
$$

When $q=1$, we have that $d: C^{0}(\mathfrak{g}) \rightarrow C^{1}(\mathfrak{g})$ is null. So, $H^{1}(\mathfrak{g})=\operatorname{Ker}(d): C^{1}(\mathfrak{g}) \rightarrow C^{2}(\mathfrak{g})$. Since for $\omega \in C^{1}(\mathfrak{g})$ and $X, Y \in \mathfrak{g}$

$$
d \omega(X, Y)=\frac{1}{2} \omega([X, Y])
$$

$\omega$ is a cocycle if, and only if, it vanishes for all elements on $[\mathfrak{g}, \mathfrak{g}]$. Thus,

$$
H^{1}(\mathfrak{g})=\mathfrak{g} /[\mathfrak{g}, \mathfrak{g}]
$$

2. We can set $V=\mathfrak{g}$ and the adjoint representation $\rho(X)(Y)=\operatorname{ad}(X, Y)=[X, Y]$ for all $X, Y \in \mathfrak{g}$. On this case the diferential operator of the cohomology $H^{\bullet}(\mathfrak{g}, \mathfrak{g})$ is given by

$$
\begin{aligned}
d(\omega)\left(X_{1}, \ldots, X_{q+1}\right)= & \sum_{i=1}^{q+1}(-1)^{i+1}\left[X_{i}, \omega\left(X_{1}, \ldots, \hat{X}_{i}, \ldots, X_{q+1}\right)\right] \\
& +\sum_{i<j}(-1)^{i+j} \omega\left(\left[X_{i}, X_{j}\right], X_{1}, \ldots, \hat{X}_{i}, \ldots, \hat{X}_{j}, \ldots X_{q+1}\right) .
\end{aligned}
$$


Example 84 (Poisson cohomology). The Poisson cohomology, discussed in Section 2.2, can be seeing as a special case of the Lie algebroid cohomology. Let $A=T^{*} M$ be the cotangent algebroid of the Poisson manifold $(M, \Pi)$ and $E=M \times \mathbb{R}$ be the trivial line bundle. Choosing the following representation

$$
\begin{aligned}
\#: \Gamma(T * M) \times C^{\infty}(M) & \rightarrow C^{\infty}(M) \\
(\omega, f) & \mapsto \#(\omega)(f)
\end{aligned}
$$

A p-cochain is nothing but a p-vector field.

Proposition 85. The differential operator $d$ of the cotangent algebroid of the Poisson manifold $(M, \Pi)$ with the above representation is given by

$$
d=d_{\Pi}=[\Pi, \cdot]
$$

Proof. For a vector $X \in \mathfrak{X}(M)$ and $\alpha, \beta \in \Gamma\left(T^{*} M\right)$ we have

$$
\begin{aligned}
d(X)(\alpha, \beta)= & \mathscr{L}_{\#(\alpha)}(X(\beta))-\mathscr{L}_{\#(\beta)}(X(\alpha))-\langle[\alpha, \beta], X\rangle \\
= & \mathscr{L}_{\#(\alpha)}(\beta)(X)+\left\langle\beta, \mathscr{L}_{\#(\alpha)}(X)\right\rangle-\mathscr{L}_{\#(\beta)}(\alpha)(X)-\left\langle\alpha, \mathscr{L}_{\#(\beta)}(X)\right\rangle \\
& -\mathscr{L}_{\#(\alpha)}(\beta)(X)+\mathscr{L}_{\#(\beta)}(\alpha)(X)+X(\Pi(\alpha, \beta)) \\
= & \left\langle\beta, \mathscr{L}_{\#(\alpha)}(X)\right\rangle-\left\langle\alpha, \mathscr{L}_{\#(\beta)}(X)\right\rangle+X(\Pi(\alpha, \beta))
\end{aligned}
$$

On the other hand,

$$
\begin{aligned}
d_{\Pi}(x)(\alpha, \beta)= & {[\Pi, X](\alpha, \beta)=\mathscr{L}_{X}(\Pi)=-\mathscr{L}_{X}(\Pi(\alpha, \beta))+\Pi\left(\mathscr{L}_{X}(\alpha \wedge \beta)\right) } \\
= & -X(\Pi(\alpha, \beta))+\Pi\left(\mathscr{L}_{X}(\alpha), \beta\right)+\Pi\left(\alpha, \mathscr{L}_{X}(\beta)\right) \\
= & -X(\Pi(\alpha, \beta))-\mathscr{L}_{X}(\alpha)(\#(\beta))+\mathscr{L}_{X}(\beta)(\#(\alpha)) \\
& =-X(\Pi(\alpha, \beta))-X(\Pi(\beta, \alpha))+\left\langle\alpha, \mathscr{L}_{X}(\#(\beta))\right\rangle+X(\Pi(\alpha, \beta))-\left\langle\beta, \mathscr{L}_{X}(\#(\alpha))\right\rangle \\
= & \left\langle\beta, \mathscr{L}_{\#(\alpha)}(X)\right\rangle-\left\langle\alpha, \mathscr{L}_{\#(\beta)}(X)\right\rangle+X(\Pi(\alpha, \beta)) .
\end{aligned}
$$

Therefore, the result holds for the case $X \in \mathfrak{X}(M)$. Now suppose the result has been proved for $A \in \mathfrak{X}^{a}(M)$, and let $X \in \mathfrak{X}(M)$. From theorem 13

$$
[\Pi, A \wedge X]=[\Pi, A] \wedge X+(-1)^{a} A \wedge[\Pi, X]
$$

On Section 7.5 from [Mackenzie 2005], Mackenzie proves that

$$
d\left(A^{X}\right)=d(A) \wedge X+(-1)^{a} A \wedge d(X)
$$

Since the result holds for $X \in \mathfrak{X}(M)$ and $A \in \mathfrak{X}^{a}(M)$, thus it also holds for $\xi \in \mathfrak{X}^{a+1}(M)$. 


\subsection{Lie groupoid}

Definition 86. A groupoid $\mathscr{G} \rightrightarrows M$ over a set $M$, called objects set, is a set $\mathscr{G}$, called set of arrows, together with:

1. Two surjective maps $s, t: \mathscr{G} \rightarrow M$, associating to each arrow g its source object $s(g)$ and its target object $t(g)$. We call them, respectively, the source and the target map.

$$
t(g) \longleftarrow s(g)
$$

2. The partial composition map, $m: \mathscr{G}_{2} \rightarrow \mathscr{G},(g, h) \mapsto g h$

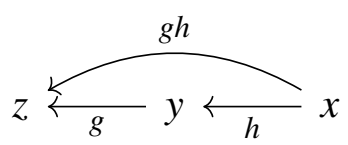

where $\mathscr{G}_{2}=\{(g, h) \in \mathscr{G} \times \mathscr{G} \mid s(g)=t(h)\}$, satisfying

a) Compatibility with $s$ e $t$ :

$$
s(g h)=s(h), \quad t(g h)=t(g) \quad \forall(g, h) \in \mathscr{G}_{(2)} .
$$

b) Associativity:

$$
\begin{aligned}
& (h g) f=h(g f) \forall f, g, h \in \mathscr{G} \text { with } s(g)=t(h) \text { e } s(h)=t(k) \text {. }
\end{aligned}
$$

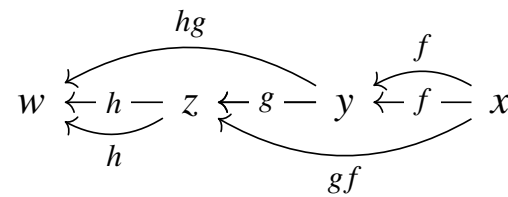

3. The inclusion map

$$
e: M \rightarrow \mathscr{G}, x \mapsto 1_{x},
$$

such that, for any $g: x \rightarrow y$,

$$
g 1_{x}=g=1_{y} g
$$

4. The inverse map,

$$
i: \mathscr{G} \rightarrow \mathscr{G}, g \mapsto g^{-1},
$$

such that $s(i(g))=t(g), t(i(g))=s(g)$, and

$$
g^{-1} g=1_{s(g)}, g g^{-1}=1_{t(g)} .
$$

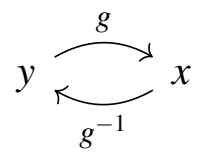


Example 87. Let $B$ be a set and $G$ be a group. We define a groupoid structure on $B \times G \times B$ as follows.

- $s, t$ are defined, respectively, as the projections onto the first and third factor;

- The identity is the map $x \mapsto(x, 1, x)$, where 1 is the group identity;

- The multiplication operation $(z, h, y)\left(y^{\prime}, g, x\right)=(z, h g, x)$, is defined only if $y^{\prime}=y$;

- The inverse map of $(y, g, x)$ is $\left(x, g^{-1}, y\right)$.

For example, every group is a groupoid over $B=\{p\}$.

Example 88. Let $B$ be a topological space. We define the space of arrows between every $x, y \in B$ as the homotopy-classes $\langle c\rangle$ of continuous maps $c: I \rightarrow B$ which endpoints are $x$ and $y$. The groupoid structure over $B$ is given by

- $s(\langle c\rangle)=c(0)$

- $t(\langle c\rangle)=c(1)$;

- $e(x)=\langle c\rangle=x$

- composition

$$
\left\langle c_{1}\right\rangle\left\langle c_{2}\right\rangle= \begin{cases}c_{1}(2 t), & 0 \geq t \leq \frac{1}{2} \\ c_{2}(2 t), & \frac{1}{2} \leq t \leq 1\end{cases}
$$

- $\langle c\rangle^{-1}=\langle c(1-t)\rangle$.

Now, we present the concept of morphism between two groupoids, $\mathscr{G} \rightrightarrows M$ and $\mathscr{G}^{\prime} \rightrightarrows M^{\prime}$.

Definition 89. Let $\mathscr{G} \rightrightarrows M$ and $\mathscr{G}^{\prime} \rightrightarrows M^{\prime}$ be groupoids. A morphism betwenn $\mathscr{G}$ and $\mathscr{G}^{\prime}$ is a pair of maps $\Phi: \mathscr{G} \rightarrow \mathscr{G}^{\prime}, \varphi: M \rightarrow M^{\prime}$, such that,

I $\varphi(s(g))=s^{\prime}(\Phi(g))$ and $\varphi(t(g))=t^{\prime}(\Phi(g))$, that is, the following diagram commutes
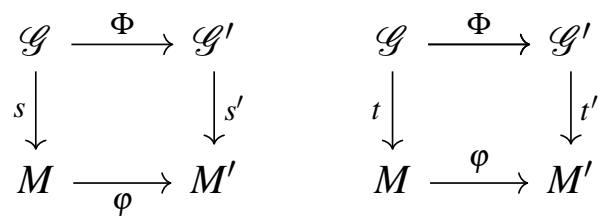

II $\Phi\left(g_{1} g_{2}\right)=\Phi\left(g_{1}\right) \Phi\left(g_{2}\right)$

for all $g, g_{1}, g_{2} \in \mathscr{G}$ with $s\left(g_{1}\right)=t\left(g_{2}\right)$. We also say that $\Phi$ is a morphism over $\varphi$.

Remark 90. The groupoid definitions is the same of a category which all the arrows are mesomorphs. The definition of groupoid morphs is a functor. 
Definition 91. A Lie groupoid is a groupoid $\mathscr{G} \rightrightarrows M$ which $\mathscr{G}$ and $M$ are smooth manifolds, the structural functions are smooth and $s, t$ are submersion.

Remark 92. It is usual to permit that the space of arrows be a non-Hausdorff manifold [Crainic and Fernandes 2011]. When it is Hausdorff it is called Hausdorff groupoid but during this monograph we assume that all groupoids are Hausdorff groupoids.

A morphism between Lie groupoids is a morphism of groupoids $(\Phi, \varphi)$ where both $\Phi$ and $\varphi$ are smooth. We say that a morphism between two Lie groupoids is a isomorphism if both $(\Phi, \varphi)$ are diffeomorphisms.

Example 93. Let $E \stackrel{\pi}{\rightarrow} M$ be a vector bundle. Let $\Phi(E)$ be the set of all isomorphisms $\eta: E_{x} \rightarrow$ $E_{y}$, for all $x, y \in M$. Then $\Phi(E)$ is a Lie groupoid over $M$.

- The source and target are given by $E_{x} \stackrel{\psi}{\rightarrow} R_{y}, s(\psi)=x$ and $t(\psi)=y$.

- The indent map of $x$ is $1_{x}=I d: E_{x} \rightarrow E_{x}$.

- The multiplication is defined by the composition of linear maps.

- The inverse of $\psi: E_{x} \rightarrow E_{y}$ is the inverse map $\psi^{-1}: E_{y} \rightarrow E_{x}$

Example 94. Let $G \times M \rightarrow M$ be a smooth action of a Lie group $G$ in a manifold $M$. We define $G \times M \rightrightarrows M$ by:

Given any arrow $(g, x) \in G \times M$

$$
s(g, x)=x, t(g, x)=g x .
$$

Let $\left(g_{2}, y\right),\left(g_{1}, y\right) \in G \times M$, such that $y=g_{1} x$, so

$$
\begin{gathered}
\left(g_{2}, y\right)\left(g_{1}, x\right)=\left(g_{2} g_{1}, x\right) . \\
(g, x)^{-1}=\left(g^{-1}, g x\right), \\
1_{x}=(x, e), \text { where } e \text { is the identity of } G .
\end{gathered}
$$

We denote $G \times M \rightrightarrows M$ by $G \ltimes M$.

Example 95. Let $\mathscr{G} \rightrightarrows M$ be a Lie groupoid. We define a Lie groupoid over $\mathscr{G}$ as

- The arrows manifold is $\mathscr{G} \times s, \mathscr{G}=\{(h, g \in \mathscr{G} \times \mathscr{G} \mid s(h)=s(g)\}$.

- The composition is defined by

$$
(h, g) \circ(g, f)=(h, f) .
$$


We define a groupoid morphism
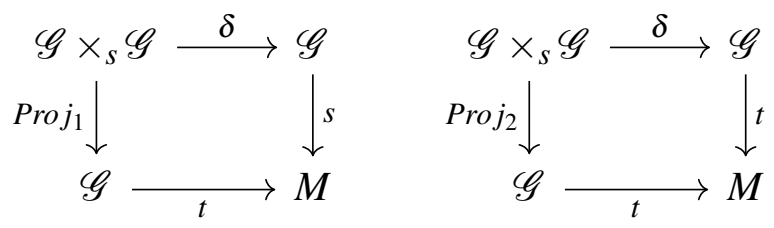

where $\delta(h, g)=h g^{-1}$.

Definition 96. For a groupoid $\mathscr{G} \rightrightarrows M$ and $x \in M$ we define

I The $s$-fiber of $x$ by $\mathscr{G}_{x}:=s^{-1}(x)$.

II The $t$-fiber of $x$ by $\mathscr{G}^{x}:=t^{-1}(x)$.

III The isotropy group at $x$ by $\mathscr{G}_{x}^{x}:=s^{-1}(x) \cap t^{-1}(x)$.

\subsubsection{The Lie algebroid of a Lie groupoid}

If $G$ is a Lie Group the vector space of left (right) invariant vector fields is a Lie subalgebra of $\mathfrak{X}(G)$. This subalgebra is finite dimensional and caries a lot of information about the Lie group. Indeed, it is isomorphic to the tangent space of the group at identity. This construction defines a functor from the category of Lie groups to the category of Lie algebras. In this section, we will show how a Lie groupoid gives rise to a Lie algebroid. To this purpose, it is necessary to generalize the idea of right-invariant vector fields.

Definition 97. Let $\mathscr{G} \rightrightarrows M$ be a Lie groupoid. Let $T^{s} \mathscr{G}$ be the vector bundle over $\mathscr{G}$ defined by

$$
T^{s} \mathscr{G}:=\operatorname{Ker}(d s) \subset T \mathscr{G} .
$$

We define $\operatorname{Lie}(\mathscr{G})$ whose fiber in $x \in M$ are the same of the tangent space in $1_{x}$ of $T^{s}(\mathscr{G})$, more precisely,the total space of the Lie algebroid would be

$$
\operatorname{Lie}(\mathscr{G}):=\left.T^{s} \mathscr{G}\right|_{M}
$$

When we try to extend the idea of right action of arrow of $\mathscr{G} \rightrightarrows M$ to $M$, this action is not everywhere defined.

Definition 98. Let $y \stackrel{g}{\leftarrow} x$ be a arrow and define the right action of $g$ by

$$
\begin{aligned}
R_{g}: s^{-1}(y) & \rightarrow s^{-1}(x) \\
h & \mapsto h g,
\end{aligned}
$$

and the differential of $R_{g}$ on $h \in \mathscr{G}$ gives us a linear isomorphism, defined by

$$
d R_{g}: T_{h} \mathscr{G} \rightarrow T_{h g} \mathscr{G}
$$


Now, we are ready to define a good notion of right invariant vector field on $\mathscr{G}$.

Definition 99. We define the space of right invariant vector fields of $\mathscr{G}$ as

$$
\mathfrak{X}_{\text {inv }}^{s}(\mathscr{G})=\left\{X \in \Gamma\left(T^{s} \mathscr{G}\right): X_{h g}=d R_{g}\left(X_{h}\right), \quad \forall(g, h) \in \mathscr{G}_{(2)}\right\} .
$$

The next proposition ensures that $\operatorname{Lie}(\mathscr{G})$ is a Lie algebroid over $M$.

Proposition 100. If $\mathscr{G} \rightrightarrows M$ is a Lie groupoid and $\mathfrak{X}_{i n v}^{s}(\mathscr{G})$ the space of right invariant vector bundles of $\mathscr{G}$, then

1. The vector bundle morphism

$$
\begin{aligned}
\Gamma(\operatorname{Lie}(\mathscr{G})) & \rightarrow \mathfrak{X}_{i n v}^{s}(\mathscr{G}) \\
\alpha & \mapsto \widetilde{\alpha}_{g}=d R_{g}\left(\alpha_{t}(g)\right)
\end{aligned}
$$

is an isomorphism.

2. $\mathfrak{X}_{\text {inv }}^{s}(\mathscr{G}) \subset \mathfrak{X}(\mathscr{G})$ is a Lie subalgebra.

3. The derivative of the target map induces a Lie algebra homomorphism $d t: \mathfrak{X}_{i n v}^{s}(\mathscr{G}) \rightarrow$ $\mathfrak{X}(M)$.

4. For all $\alpha, \beta \in \Gamma(A)$ and for any $f \in C^{\infty}$.

$$
[\alpha, f \beta]=f[\alpha, \beta]+d t(\alpha)(f) \beta
$$

Thanks to the above preposition, the vector bundle $\operatorname{Lie}(\mathscr{G})$ is a Lie algebroid over $M$ whose the anchor map is the diferential of the target map, \# =dt, and the bracket is given by the restriction of the vector fields bracket in $T \mathscr{G}$ to $\mathfrak{X}_{\text {inv }}^{s}(\mathscr{G})$.

Example 101. When the groupoid is a Lie group, $\operatorname{Lie}(\mathscr{G})$ is the Lie algebra associated to $\mathscr{G}$.

Definition 102. When a Lie algebroid $(A,[\cdot, \cdot], \#) \cong \operatorname{Lie}(\mathscr{G})$, for some Lie groupoid, we say that $\mathscr{G}$ integrates $A$.

Proposition 103. Let $(A,[\cdot, \cdot], \#)$ be a Lie algebroid and $x_{0} \in A$ a fixed point. If $U$ is a neighborhood of $x_{0}$ which integrates to a Lie groupoid $\mathscr{G}$, then the fiber of the source map $s: \mathscr{G} \rightrightarrows U$ above $x_{0}$ is a Lie group integranting $\mathfrak{g}_{x_{0}}$.

\subsection{Integrability problem}

We have seen in the previous section how to construct a Lie algebroid, starting from a Lie groupoid. A natural question one is led to ask is: under what conditions is it possible to do 
the opposite process, i. e., when is it possible to get a Lie groupoid that integrates a given Lie algebroid?

In the case of Lie groups and Lie algebras, the answer is given by the Lie's third theorem. A classic and famous proof is obtained as a consequence of the Ado's theorem [Tao 2011]. A different approach is presented in [Duistermaat and Kolk 1999], which is based on a path space approach to construct the Lie group integrating the original Lie algebra.

The integrability problem of Lie algebroids was solved by Marius Crainic and Rui Loja Fernandes both in the general case and for the Poisson one, and this was presented in [Crainic and Fernandes 2003] and [Crainic and Fernandes 2004], respectively. Adapting the idea used by Duistermaat and Kolk for the Lie's third theorem, Crainic and Fernandes showed that it is possible to define a groupoid, associated with a general Lie algebroid, called Weinstein groupoid. This section presents a brief discussion of the Lie third theorem for Lie algebroids. We followed the construction of [Crainic and Fernandes 2011], to which we refer the reader for further details.

To define this groupoid it is necessary to define the concept of $A$-path and homotopy of A-path.

Definition 104. Let $(A,[\cdot, \cdot], \#)$ be a Lie algebroid. We call a $C^{\infty}$ curve $a: I \rightarrow A$ an $A$-path if

$$
\# a(t)=\frac{d}{d t}(\pi(a(t))
$$

where $\pi(a(t))$ is the base path of $a(t)$. We denote the space of $A$-paths, endowed with $C^{1}$ topology, by $P(A)$.

The notion of $A$-path is summarized by the diagram bellow

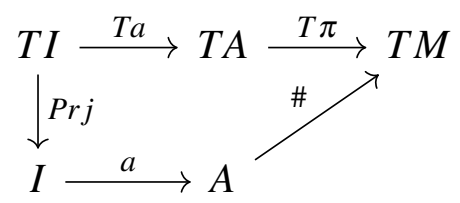

Let $\nabla$ be an usual $T M$-connection on $A$. We can associate a contravariant $A$-connection, denoted by $\bar{\nabla}$, as

$$
\bar{\nabla}_{\alpha} \beta=\nabla_{\# \beta} \alpha+[\alpha, \beta] .
$$

Proposition 105. Let $(A,[\cdot, \cdot], \#)$ be a Lie algebroid and $a_{\varepsilon}: I \times I \rightarrow A$ a variation of $A$-paths. If $\nabla$ is a $T M$ - connection on $A$ with torsion $T_{\nabla}$, then the solution $b=b(\varepsilon, t)$ of the differential equation

$$
\partial_{t} b-\partial_{\varepsilon} a=T_{\nabla}(a, b), b(\varepsilon, 0)=0,
$$

does not depend on $\nabla$. Moreover, $\# b=\frac{d}{d \varepsilon}(\pi(a))$.

So, we define the homotopic of $A$-paths as: 
Definition 106. We say that two $A$-paths $a_{0}$ and $a_{1}$ are homotopic if there exist a variation $a_{\varepsilon}$ such that the function $b$, defined on the proposition above, satisfies $b(\varepsilon, 1)=0$.

The space of A-paths is a foliated manifold where two paths $a_{0}$ and $a_{1}$ belong to the same leaf if they are homotopic. We define the Weinstein groupoid, $\mathscr{G}(A)$, as a set by the leaf space of the foliation above. The composition map is given by the concatenation of paths and the structural maps are given by $s(a)=\pi(a(0))$ and $t(a)=\pi(a(1))$.

Given a Lie algebroid, it is always possible to define the corresponding Weinstein groupoid. However, it is not always true that the Weinstein groupoid admits a structure of Lie groupoid, i. e., sometimes it is impossible to provide $\mathscr{G}(A)$ with a smooth structure that makes it into a Lie groupoid. Finding a necessary and sufficient condition for the Weinstein groupoid to admit a structure of a Lie groupoid is the main result of [Crainic and Fernandes 2003].

\subsection{Lie groupoid cohomology}

This section is based on [Crainic 2003]. We denote by $\mathscr{G}(p)$ the space of $p$-composable strings $x \stackrel{g_{1}}{\longleftarrow} \stackrel{g_{2}}{\longleftarrow} \ldots \stackrel{g_{p}}{\longleftarrow} y$.

Definition 107. A left action of $\mathscr{G} \rightrightarrows M$ on a manifold $X$ consists of a smooth map $\mu: X \rightarrow M$, called moment map of the action, together with a smooth map

$$
\begin{aligned}
\mathscr{G} \times{ }_{M} X & \rightarrow X \\
(g, x) & \mapsto g x,
\end{aligned}
$$

where $\mathscr{G} \times_{M} X=\{(g, x) \in \mathscr{G} \times X \mid s(g)=\mu(x)\}$ and such that, for all $(g, x) \in \mathscr{G} \times{ }_{M} X$,

$$
\mu(g x)=t(g)
$$

and which satisfies the usual identities of an action, i. e.,

i $g(h x)=(g h) x$, for all $g, h \in \mathscr{G}$ and $X \in X$ for which it is defined.

ii $1_{\mu(x)} x=x$, for all $x \in X$

we call $X$ left $\mathscr{G}$-space.

Definition 108. A left representation of a Lie groupoid $\mathscr{G} \rightrightarrows M$ is a vector bundle proj: $E \rightarrow M$, endowed with an action of $\mathscr{G}$ whose moment map is precisely proj and which is fiber-wise linear.

Definition 109. An $E$-valued differentiable $p$-cochain on $\mathscr{G}$ is a smooth map $c$ which associates to a string an element $c\left(g_{1}, \ldots, g_{p}\right) \in E_{x}$. We denote the space of $E$-valued $p$-cochain by $C_{d}^{p}(\mathscr{G}, E)$. 
We can define a differential operator $d: C_{d}^{p}(\mathscr{G}, E) \rightarrow C_{d}^{p+1}(\mathscr{G}, E)$

$$
\begin{aligned}
d(c)\left(g_{1}, \ldots, g_{p}, g_{p+1}\right)= & g_{1} c\left(g_{2}, \ldots, g_{p}\right) \\
& +\sum_{i=1}^{p}(-1)^{i} c\left(g_{1}, \ldots, g_{i} g_{i+1}, \ldots, g_{p+1}\right)+(-1)^{p+1} c\left(g_{1}, \ldots, g_{p}\right) .
\end{aligned}
$$

Definition 110. We define the differentiable cohomology of $\mathscr{G}$ with coefficients in $E, H_{d}^{*}(\mathscr{G}, E)$, as the differential cohomology of the complex $\left(C_{d}^{*}(\mathscr{G}, E), d\right)$. When $E$ is the trivial line bundle, we use the notation $C_{d}^{*}(\mathscr{G})$ and $H^{*}(\mathscr{G})$.

Proposition 111. For any proper Lie groupoid $\mathscr{G}$, and $E$, for all $k \geq 1$,

$$
H_{d}^{K}(\mathscr{G}, E)=0
$$

Let $P$ be a right $\mathscr{G}$-space. We call it proper if the map

$$
\begin{aligned}
\mathscr{G} \times{ }_{M} P & \rightarrow P \times P \\
(g, p) & \mapsto(g p, p)
\end{aligned}
$$

is proper, i. e., if the inverse images of compact subsets are compact.

Theorem 112. Let $\mathscr{G} \rightrightarrows M$ be a $s-$ connected Lie groupoid, and let $E$ be a representation of $\mathscr{G}$. Then there exists a map

$$
\Phi: H_{d}^{p}(\mathscr{G}, E) \rightarrow H_{\mathscr{G}}^{p}(\operatorname{Lie}(\mathscr{G}), E),
$$

which is compatible with the product structures. Moreover, if the $s$-fibers are homologically $n$-connected, then $\Phi$ is an isomorphism in degrees $p \leq n$, and it is injective in degree $p=n+1$.

Example 113 (Lie Group Cohomology). Let $G$ be a Lie group and $V$ a vector space. A representation of $G$ on $V$ is a smooth group homomorphism $\rho: G \rightarrow G L(V)$, where $G L(V)$ is the general linear group of all invertible linear transformations of $V$.

Similar to the Lie algebra cohomology, given a Lie group $G$ and a representation $\rho: G \rightarrow$ $G L(V)$, we define the cochains as

$$
C^{q}(G, V)=\left\{c: G^{q} \rightarrow V \mid c \text { is smooth }\right\}
$$

We define $d: C^{q}(G, V) \rightarrow C^{q+1}(G, V)$ by

$$
\begin{aligned}
(d \omega)\left(g_{0}, \ldots, g_{q}\right)= & \rho\left(g_{0}\right) \omega\left(g_{1}, \ldots, g_{q}\right)+\sum_{i=0}^{q-1}(-1)^{i+1} \omega\left(g_{0}, \ldots, g_{i} g_{i+1}, \ldots, g_{q}\right) \\
& +(-1)^{q+1} \omega\left(g_{0}, \ldots, g_{k-1}\right) .
\end{aligned}
$$

Then, the Lie group cohomology is defined as follows. 
Definition 114. Given a Lie group $G$ with a representation $\rho: G \rightarrow G L(V)$. Using the previous definition of $C^{q}(G, V)$ and $d: C^{q}(G, V) \rightarrow C^{q+1}(G, V)$, we define the Lie group cohomology with coeffcients in $V$ by

$$
H^{q}(G, V):=\frac{\operatorname{Ker}\left(d: C^{q}(G, V) \rightarrow C^{q+1}(G, V)\right)}{\operatorname{Im}\left(d: C^{q-1}(G, V) \rightarrow C^{q}(G, V)\right)} .
$$

When $V=\mathbb{R}$ and $\rho$ is trivial, we denote $C^{q}(G, \mathbb{R})$ and $H^{q}(G, \mathbb{R})$ only by $C^{q}(G)$ and $H^{q}(G)$.

In [Est 1953, Est 1953], Van Est presented the following result that permits us to relate Lie group cohomology with Lie algebra cohomology.

Theorem 115 (Van Est). Let $G$ be a $n$-connected Lie Group and $\rho: G \rightarrow G L(v)$ a representation. If $\mathfrak{g}$ is the Lie algebra of $G$, then $H^{i}(G, V)$ is isomorphic with $H^{i}(\mathfrak{g}, V)$ for all $i=0,1, \ldots, m$. 

CHAPTER

4

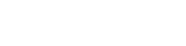

\section{CONN'S LINEARIZATION THEOREM}

Let $(M, \Pi)$ be a Poisson manifold and let $x_{0} \in M$ such that $\Pi\left(x_{0}\right)=0$. Let $\Pi=\Pi^{1}+$ $\Pi^{2}+\ldots$ be the Taylor expansion of $\Pi$ with respect to a local coordinate centered on $x_{0}$. From the condition that the Schouten bracket of $\Pi$ with itself vanishes, one deduces

$$
\begin{aligned}
0 & =[\Pi, \Pi]=\left[\sum_{k} \Pi^{k}, \sum_{k} \Pi^{k}\right] \\
& =\left[\Pi^{1}, \Pi^{1}\right]+2\left[\Pi^{1}, \Pi^{2}\right]+\left(2\left[\Pi^{1}, \Pi^{3}\right]+\left[\Pi^{2}, \Pi^{2}\right]\right)+\cdots
\end{aligned}
$$

Since every coefficient on the above expansion is a polynomial function of the local coordinates, considering the terms of same degree, we get

$$
\begin{aligned}
& {\left[\Pi^{1}, \Pi^{1}\right]=0,} \\
& {\left[\Pi^{1}, \Pi^{2}\right]=0,} \\
& 2\left[\Pi^{1}, \Pi^{3}\right]+\left[\Pi^{2}, \Pi^{2}\right]=0, \\
& \text { and so on } \ldots
\end{aligned}
$$

So, by $\left[\Pi^{1}, \Pi^{1}\right]=0$, the linear term of the expansion is a Poisson structure.

Our interest is to study the case in which $\Pi^{1}$ is non-null. According to equation (2.7.1) we can notice that $\Pi^{(1)}$ is the linear Poisson structure associated to the isotropy Lie algebra $\mathfrak{g}_{x_{0}}$.

It is always possible to truncate the series expansion and take an approximation of a bivector field by a linear one. But now, we may ask if there is any condition for a Poisson structure to be locally isomorphic to the linear approximation. The following definition summarizes the core of the linearization problem.

Definition 116. We say that $\Pi$ is locally linearizable if there is a locally defined diffeomorphism $\Phi$ such that $\Phi_{*} \Pi=\Pi^{(1)}$. 
T heorem 118 gives some conditions to the isotropy Lie algebra to carry all the information, up to local isomorphisms, about the Poisson structure near $x_{0}$ such that $\Pi\left(x_{0}\right)=0$. The following definition formalizes this idea.

Definition 117. A finite-dimensional Lie algebra $\mathfrak{g}$ is called non-degenerate if every Poisson structure $\Pi$, which vanishes at a point $x_{0}$ and whose $\Pi^{(1)}$ is the linear Poisson structure corresponding to $\mathfrak{g}$, is linearizable.

In [Weinstein 1983], Alan Weinstein proposed the following problem: how to classify the Lie algebras that are non-degenerate? In that paper, Weinstein conjectured that every compact semi-simple Lie algebra is non-degenerate. This claim was proved by Conn using non-trivial analytic arguments [Conn 1985].

Recently, a more geometric approach to Conn's theorem was presented by Marius Crainic and Rui Loja Fernandes on [Crainic and Fernandes 2011].

The goal of this chapter is to present a discussion, based on [Crainic and Fernandes 2011] as the main reference, about the proof of the following theorem.

Theorem 118 (Conn). Let $(M, \Pi)$ be a Poisson manifold and $x_{0} \in M$ such that $\Pi\left(x_{0}\right)=0$. If the isotropy Lie algebra $\mathfrak{g}_{x}$ is semi-simple of compact type, then $\Pi$ is linearizable around $x_{0}$.

\subsection{Compact Lie Algebras}

The studies of this section is presented in [Duistermaat and Kolk 1999].

Definition 119. For every Lie algebra $\mathfrak{g}$, we can associate a bilinear form, called Killing form, defined by

$$
k(x, y)=\operatorname{tr}(\operatorname{adX} \circ a d Y), X, Y \in \mathfrak{g} .
$$

Corollary 120 (3.6.3 [Duistermaat and Kolk 1999]). Let $\mathfrak{g}$ be a finite-dimensional Lie algebra. Then the following conditions are equivalent:

- $\mathfrak{g}$ is compact and semi-simple.

- $\mathfrak{g}$ is compact and its center is zero.

- $\mathfrak{g}$ has a negative definite Killing form.

- Every connected Lie group with Lie algebra isomorphic to $\mathfrak{g}$ is compact. In any of these cases, $\mathfrak{g}=[\mathfrak{g}, \mathfrak{g}]$.

In [Dufour and Zung 2006] the next theorem is presented.

Theorem 121 (Whitehead). If $\mathfrak{g}$ is semi-simple and $V$ is a finite-dimensional $\mathfrak{g}$-module, then $H^{1}(\mathfrak{g}, V)=H^{2}(\mathfrak{g}, V)=0$. 


\subsection{Reduction to a cohomological condition}

By a Poisson version of Morse's path method it is possible to find a sufficient condition to a Poisson structure to be linearizable around $x_{0}$. This condition involves only the local cohomology of the Poisson manifold and the cohomology of the isotropy Lie algebra at $x_{0}$.

Proposition 122. Let $(M,\{\cdot, \cdot\})$ be a Poisson Manifold such that $\Pi$ vanishes at $x_{0}$. Assume that the Lie algebra cohomology groups $H^{1}\left(\mathfrak{g}_{x_{0}}\right)=H^{1}\left(\mathfrak{g}_{x_{0}}, \mathfrak{g}_{x_{0}}\right)=0$. If $H_{\Pi}^{1}\left(M, x_{0}\right)=0$, then $\{\cdot, \cdot\}$ is linearizable at $x_{0}$.

Proof. Since this is a local fact, without loss of generality, one can assume that $M=\mathbb{R}^{n}$ and $x_{0}=0$. We define a path of Poisson structures by

$$
\Pi_{t}(x)=\frac{1}{t} \Pi(t x), t \in[0,1] .
$$

We will show that there exists a vector field $X$ in an open neighborhood of 0 whose linearization vanishes at the origin and

$$
\mathscr{L}_{X}(\Pi)=-\dot{\Pi}_{1}
$$

Indeed, taking the time derivative of $\left[\Pi_{t}, \Pi_{t}\right]=0$, at $t=1$, one has

$$
\left.\frac{d}{d t}\left[\Pi_{t}, \Pi_{t}\right]\right|_{t=1}=\left[\dot{\Pi}_{1}, \Pi_{1}\right]+\left[\Pi_{1}, \dot{\Pi}_{1}\right]=\left[\Pi, \dot{\Pi}_{1}\right]-\left(-1^{(2-1)(2-1)}\right)\left[\Pi, \dot{\Pi}_{1}\right]=0
$$

so $d_{\Pi}\left(\dot{\Pi}_{1}\right)=\left[\Pi, \dot{\Pi}_{1}\right]=0$, and since $H_{\Pi}^{2}(M, 0)=0$, there is an open neighborhood $U$ of the origin and a vector field on $U$ such that

$$
\dot{\Pi}_{1}=d_{\Pi} Y=-\mathscr{L}_{Y}(\Pi)
$$

Since $\dot{\Pi}_{1}=0$ at $x_{0}$, if we evaluate both sides of the above expression on a pair of 1 -forms $\alpha, \beta$

$$
Y_{x_{0}}([\alpha, \beta])=\left.\mathscr{L}_{Y}(\Pi)(\alpha, \beta)\right|_{x=0}=\left.\dot{\Pi}_{1}(\alpha, \beta)\right|_{x_{0}}=0 .
$$

By hypothesis, $H^{1}\left(\mathfrak{g}_{x_{0}}\right)=0$, so $\left[\mathfrak{g}_{x_{0}}, \mathfrak{g}_{x_{0}}\right]=\mathfrak{g}_{x_{0}}$ and then, we have that $Y_{x_{0}}=0$.

Let $Y_{0}$ be the linearization of $Y$ at the origin. Since $\dot{\Pi}_{1}=0$ has zero linearization at the origin, if we compare the linear terms of the both sides at $x_{0}$

$$
d_{\Pi_{x_{0}}} Y_{0}=0
$$

Hence, $Y_{0}$ is a 1-cocycle for the adjoint representation of $\mathfrak{g}_{x_{0}}$. Since $H^{1}\left(\mathfrak{g}_{x_{0}}, \mathfrak{g}_{x_{0}}\right)=0$, there is $v \in \mathfrak{g}_{x_{0}}$ such that $Y_{0}=d_{\Pi_{x_{0}}} v$. Since $\left(d_{\Pi}\right)^{2}=0$, if we set $X=Y-d_{\Pi} v$ we have that

$$
\mathscr{L}_{X}(\Pi)=0
$$


and the linearization of $X$ at the origin is zero.

Now we can construct a time dependent vector field defined by $X_{t}(x):=\frac{1}{t^{2}} X(t x)$. So, by 4.2.1,

$$
\mathscr{L}_{X_{t}}\left(\Pi_{t}\right)=-\dot{\Pi}_{t}
$$

Let $\Phi_{t}$ be the flow in the $X_{t}$ such that it is defined in some neighborhood $V$ of the origin for $t \in[0,1]$. So

$$
\frac{d}{d t}\left(\Phi_{t}\right)_{*} \Pi_{t}=(\Phi)_{*}\left(\mathscr{L}_{X_{t}}\left(\Pi_{t}\right)+\frac{d \Pi_{t}}{d t}\right)=0 .
$$

So, $\left(\Phi_{t}\right)_{*} \Pi_{t}$ is constant in relation to $t$ and $\Phi_{1}$ is the desired diffeomorphism.

Thus, we have a cohomological condition that implies in the linearization. By the Whitehead theorem (121), the condition of semi-simplicity implies that $H^{1}\left(\mathfrak{g}_{x_{0}}\right)=H^{1}\left(\mathfrak{g}_{x_{0}}, \mathfrak{g}_{x_{0}}\right)=$ 0 . With the purpose of trying to change this condition to one that refers to the isotropy Lie algebra in $x_{0}$ only, we will go back to the integrability problem for associated Lie algebroid of $M$.

Theorem 123. Let $(M, \Pi)$ be a Poisson manifold, $x_{0} \in M$ such that $\mathfrak{g}_{x_{0}}$ is semi-simple of compact type . If some neighborhood of $x_{0}$ is integrable by a Lie groupoid with 1-connected s-fibers, then

$$
H_{\Pi}^{2}\left(M, x_{0}\right)=0
$$

Now we reduce the proof of Coinn's linearization theorem to local integrability around $x_{0}$. On [Crainic and Fernandes 2004], Crainic and Fernandes, show the relation of the integrability of Poisson manifolds and symplectic realization.

Theorem 124. Let $(M, \Pi)$ be a Poisson manifold and $x_{0} \in M$ a fixed point. If a neighborhood $U$ of $x_{0}$ admits a symplectic realization $v: S \rightarrow U$ with the property that $v^{-1}\left(x_{0}\right)$ is simply connected and compact, then there exists a neighborhood of $x_{0}$ which is integrable by a Hausdorff Lie groupoid with simple connected s-fibers.

The following theorem shows that, if the hypotheses of Conn's linearization theorem are true, the hypotheses of the previous theorem are also true. Thus, the statements of Conn's linearization theorem holds.

Theorem 125. Let $(M, \Pi)$ be a Poisson manifold, $x_{0} \in M$ a fixed point, such that, the isotropy Lie algebra $\mathfrak{g}$ at $x_{0}$ is semi-simple of compact type with associated simply connected Lie group G. Then there exists a symplectic realization $v: S \rightarrow U$ of some open neighborhood $U$ of $x_{0}$ such that $v^{-1}\left(x_{0}\right)=G$.

Example 126 (A non-linearizable Poisson structure). Consider the linear Poisson structure on $\mathfrak{s l}^{*}(2) \simeq \mathbb{R}^{3}$ given by $\{x, y\}_{0}=-z,\{y, z\}_{0}=x,\{z, x\}_{0}=y$. The function $f(x, y, z)=x^{2}+y^{2}-z^{2}$ is in the center of $\{\cdot, \cdot\}$. So, $f$ is a Casimir function whose symplectic leaves are hyperboloids with vertex in the origin of $\mathbb{R}^{3}$. Let $g(f)$ be such that $g(f)=0$ if $f \leq 0$ and $g(f)>0$ if 
$f>0$. Consider the perturbation to $\{\cdot, \cdot\}_{0}$ given by $\{x, y\}=-z,\{y, z\}=x-\frac{y}{x^{2}+y^{2}} g(f(x, y, z))$, $\{z, x\}=y+\frac{x}{x^{2}+y^{2}} g(f(x, y, z))$.

The Hamiltonian vector field

$$
X_{z}=-\left(y+\frac{x}{x^{2}+y^{2}} g(f(x, y, z))\right) \frac{\partial}{\partial x}+\left(x-\frac{y}{x^{2}+y^{2}} g(f(x, y, z))\right) \frac{\partial}{\partial y} .
$$

Since $-y \frac{\partial}{\partial x}+x \frac{\partial}{\partial y}$ is a periodic vector field, the integral curves of $X_{z}$ in the region out of the cone $\left\{x^{2}+y^{2}-z^{2}=0\right\}$ will spiral in the direction of the cone. Thus, while the symplectic leaves of $\{\cdot, \cdot\}$ are closed, the symplectic leaves of $\{\cdot, \cdot\}$ in the region contain the cone $\left\{x^{2}+y^{2}-z^{2}=0\right\}$ in their closure.

On [Weinstein 1987], Weinstein presented the following theorem.

Theorem 127. Let $\mathfrak{g}$ be a semi-simple Lie algebra of non-compact type with rank at least two. Then $\mathfrak{g}$ is degenerate.

The reader can refer to [Dufour and Zung 2006, Weinstein 1987] for a more detailed discussion.

Lemma 128. Let $X, Y$ be two Poisson vector fields of a Poisson manifold $(M, \Pi) . \Pi+X \wedge Y$ is a Poisson structure if, and only if, $X, Y$ and $[X, Y]$ are linearly dependent or $[X, Y]=0$.

Proof. Calculating the Schouten bracket of $\Pi+X \wedge Y$ with itself, we have

$$
\begin{aligned}
{[\Pi+X \wedge Y, \Pi+X \wedge Y] } & =[\Pi, \Pi]+2[\Pi, X \wedge Y]+[X \wedge Y, X \wedge Y] \\
& =2([\Pi, X] \wedge Y-X \wedge[\Pi, Y])+[X \wedge Y, X \wedge Y] \\
& =[X \wedge Y, X] \wedge Y-X \wedge[X \wedge Y, Y] \\
& =X \wedge[Y, X] \wedge Y-X \wedge[X, Y] \wedge Y \\
& =2 X \wedge[Y, X] \wedge Y .
\end{aligned}
$$

The previous expression is equal to zero just when $[X, Y]=0$ or $X, Y$, and $[X, Y]$ are linearly dependent.

The following theorem says that for any non-compact real semi-simple Lie algebra of real rank at least 2 , it is possible to find two vector fields satisfying the conditions of the lemma above.

Theorem 129. Let $\mathfrak{g}$ be a real semi-simple Lie algebra of real rank $r \geq 1$. Then for the linear Poisson structure $\Pi_{l i n}$ on the dual space $\mathfrak{g}^{*}$, there are $r$ smooth Poisson vector fields $X_{1}, \ldots, X_{r}$ which are flat at the origin and which commute pair-wise, and such that, in an open subset of $\mathfrak{g}^{*}$ whose closure contains the origin. These vector fields span a $r$-dimensional distribution whose intersection with characteristic distribution is trivial. 
So, taking two vector fields, $X$ and $Y$, given by the theorem above, the Poisson structure $\Pi=\Pi_{\text {lin }}+X \wedge Y$ has linear part equal to $\Pi_{\text {lin }}$. Since $X$ and $Y$ are linearly independent and are traversal to the symplectic leaves of $\Pi_{l i n}$, the rank of $\Pi$ is equal to the rank of $\Pi_{l i n}+2$. Thus, $\Pi_{\text {lin }}$ can not be isomorphic to $\Pi$. 
ABRAHAM, R.; MARSDEN, J.; RATIU, T. Manifolds, Tensor Analysis, and Applications. [S.1.]: Springer New York, 1993. (Applied Mathematical Sciences). Citation on page 30.

CHEVALLEY, C.; EILENBERG, S. Cohomology theory of Lie groups and Lie algebras. Trans. Amer. Math. Soc., v. 63, p. 85-124, 1948. Citation on page 59.

CONN, J. F. Normal forms for smooth poisson structures. Annals of Mathematics, Annals of Mathematics, v. 121, n. 3, p. 565-593, 1985. Citation on page 72.

COURANT, T. J. Dirac manifolds. American mathematical society, v. 319, n. 2, p. 631-661, 1990. Citation on page 54.

CRAINIC, M. Differentiable and algebroid cohomology, van est isomorphisms, and characteristic classes. Commentarii Mathematici Helvetici, v. 78, n. 4, p. 681-721, 2003. Citations on pages 58 and 67.

CRAINIC, M.; FERNANDES, R. L. Integrability of lie brackets. Annals of Mathematics, v. 157, n. 2, p. 575-620, 2003. Citations on pages 66 and 67.

. Integrability of poisson brackets. Journal of differential geometry, v. 66, n. 1, p. 71-137, 2004. Citations on pages 46, 66, and 74 .

A geometric approach to conn's linearization theorem. Annals of Mathematics, v. 173, n. 2, p. 1121-1139, 2011. Citations on pages 11, 13, 18, and 72.

. Lectures on integrability of lie brackets. In: Lectures on Poisson geometry. [S.1.]: Geom. Topol. Publ., Coventry, 2011. (Geom. Topol. Monogr., v. 17), p. 1-107. Citations on pages $52,59,63$, and 66 .

DUFOUR, J.; ZUNG, N. Poisson Structures and Their Normal Forms. [S.1.]: Birkhäuser Basel, 2006. (Progress in Mathematics). Citations on pages 25, 29, 36, 39, 40, 43, 46, 54, 72, and 75 .

DUISTERMAAT, J.; KOLK, J. Lie Groups. [S.1.]: Springer Berlin Heidelberg, 1999. (Universitext). Citations on pages 66 and 72.

EST, W. V. Group cohomology and lie algebra cohomology in lie groups. i. Indagationes Mathematicae (Proceedings), v. 56, p. 484 - 492, 1953. Citation on page 69.

Group cohomology and lie algebra cohomology in lie groups. ii. Indagationes Mathematicae (Proceedings), v. 56, p. 493 - 504, 1953. Citation on page 69.

FERNANDES, R. L.; MARCUT, I. Lectures on Poisson Geometry. 2015. Available: <https: //faculty.math.illinois.edu/ ruiloja/Math595/book.pdf>. Accessed: 25/05/2018. Citation on page 32. 
LAURENT-GENGOUX, C.; PICHEREAU, A.; VANHAECKE, P. Poisson Structures. [S.1.]: Springer Berlin Heidelberg, 2012. (Grundlehren der mathematischen Wissenschaften). Citations on pages $33,37,41$, and 43 .

MACKENZIE, K. C. H. General Theory of Lie Groupoids and Lie Algebroids. [S.1.]: Cambridge University Press, 2005. Citation on page 60.

MARSDEN, J.; RATIU, T. Introduction to Mechanics and Symmetry: A Basic Exposition of Classical Mechanical Systems. [S.1.]: Springer New York, 2002. (Texts in Applied Mathematics). Citations on pages 30 and 45.

MEINRENKEN, E. INTRODUCTION TO POISSON GEOMETRYLECTURE NOTES, WINTER 2017. 2017. Available: <http://www.math.toronto.edu/mein/teaching/MAT1341_ PoissonGeometry/Poisson8.pdf>. Accessed: 20/02/2019. Citation on page 54.

MOERDIJK, I.; MRCUN, J. Introduction to Foliations and Lie Groupoids. [S.1.]: Cambridge University Press, 2003. (Cambridge Studies in Advanced Mathematics). Citation on page 39.

SILVA, A. da; WEINSTEIN. Geometric Models for Noncommutative Algebras. [S.1.]: American Mathematical Society, 1999. Citation on page 46.

TAO, T. Ado's theorem. 2011. Available: <https://terrytao.wordpress.com/2011/05/10/ ados-theorem>. Accessed: 30/10/2017. Citation on page 66 .

VAISMAN, I. Lectures on the Geometry of Poisson Manifolds. [S.1.]: Birkhäuser Basel, 2012. (Progress in Mathematics). Citations on pages 25, 26, 39, 40, 43, and 46.

WARNER, F. Foundations of Differentiable Manifolds and Lie Groups. [S.1.]: Springer, 1983. (Graduate Texts in Mathematics). Citations on pages 40 and 44.

WEINSTEIN, A. The local structure of poisson manifolds. J. Differential Geom., Lehigh University, v. 18, n. 3, p. 523-557, 1983. Citations on pages 37, 46, and 72.

. Poisson geometry of the principal series and nonlinearizable structures. Journal of Differential Geometry - J DIFFEREN GEOM, v. 25, p. 55-73, 01 1987. Citation on page 75 .

. Poisson geometry. Differential Geometry and its Applications, v. 9, n. 1, p. 213 - 238, 1998. Citation on page 19. 


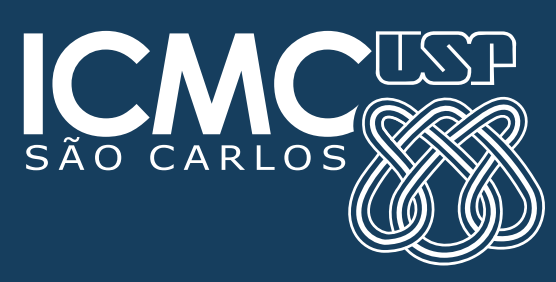

